\section{OPEN ACCESS}

Edited by:

Anthony Isles,

Cardiff University, United Kingdom

Reviewed by:

Fernando Calahorro, University of Southampton,

United Kingdom

Shohei Mitani,

Tokyo Women's Medical University,

Japan

*Correspondence:

Jean-Jacques Remy

jean-jacques.remy@inrae.fr

Specialty section:

This article was submitted to

Developmental Epigenetics,

a section of the journal

Frontiers in Cell and Developmental

Biology

Received: 10 June 2020 Accepted: 12 October 2020 Published: 30 October 2020

Citation:

Fernandes De Abreu DA

Salinas-Giegé T, Drouard L and Remy J-J (2020) Alanine tRNAs Translate Environment Into Behavior

in Caenorhabditis elegans.

Front. Cell Dev. Biol. 8:571359. doi: 10.3389/fcell.2020.571359

\section{Alanine tRNAs Translate Environment Into Behavior in Caenorhabditis elegans}

\author{
Diana Andrea Fernandes De Abreu' ${ }^{1}$, Thalia Salinas-Giegé ${ }^{2}$, Laurence Drouard ${ }^{2}$ and \\ Jean-Jacques Remy ${ }^{1 *}$ \\ ${ }^{1}$ Genes, Environment, Plasticity, Institut Sophia Agrobiotech ISA UMR CNRS 7254, INRAE 1355, Université Nice Côte \\ d'Azur, Sophia-Antipolis, France, ${ }^{2}$ Institut de Biologie Moléculaire des Plantes-CNRS, Université de Strasbourg, Strasbourg, \\ France
}

Caenorhabditis elegans nematodes produce and maintain imprints of attractive chemosensory cues to which they are exposed early in life. Early odor-exposure increases adult chemo-attraction to the same cues. Imprinting is transiently or stably inherited, depending on the number of exposed generations. We show here that the Alanine tRNA (UGC) plays a central role in regulating $C$. elegans chemo-attraction. Naive worms fed on tRNA Ala (UGC) purified from odor-experienced worms, acquire odorspecific imprints. Chemo-attractive responses require the tRNA-modifying Elongator complex sub-units 1 (elpc-1) and 3 (elpc-3) genes. elpc-3 deletions impair chemoattraction, which is fully restored by wild-type tRNA Ala (UGC) feeding. A stably inherited decrease of odor-specific responses ensues from early odor-exposition of elpc-1 deletion mutants. tRNA Ala (UGC) may adopt various chemical forms to mediate the cross-talk between innately-programmed and environment-directed chemo-attractive behavior.

Keywords: epitranscriptome, tRNA, behavior, plasticity, chemoattraction

\section{INTRODUCTION}

Although parental adaptation to different environmental challenges can be transmitted to future generations, the mechanisms by which external signals are translated into heritable information are largely unknown (Liberman et al., 2019).

Caenorhabditis elegans worms keep a life-term memory of attractive olfactory cues to which they were exposed during the first larval stage (Remy and Hobert, 2005). Early odor-exposure results in a significant enhancement of odor-specific chemo-attraction at the adult stage. Such olfactory imprinting can be inherited either transiently by a single generation or stably over generations after at least five consecutive generations were exposed to the same cue (Remy, 2010).

Non-coding RNAs have previously been implicated in the memory and transgenerational transmission of different environmental information in the C. elegans nematode (Juang et al., 2013; Hall et al., 2013; Rechavi et al., 2014; Posner et al., 2019) and in the mouse (Gapp et al., 2014; Grandjean et al., 2015; Benito et al., 2018).

In this work, we found that a single Alanine tRNA molecule plays an essential role in regulating both the innately expressed and the environment modulated chemo-attractive responses in C. elegans. We first observed that naive unexposed worms acquire an odor-specific imprinting after 
being fed on RNA extracted from odor-exposed animals. Biochemical fractionation of RNAs led to identify transfer RNAs (tRNAs) containing fractions as the imprinting transmission medium. Using tRNA-specific probes, we found that among all C. elegans tRNA molecules, only the Alanine tRNA with the UGC anticodon, tRNA Ala (UGC), transfers odor-specific imprints to naive worms via feeding. The highly purified tRNA ${ }^{\text {Ala }}$ (UGC) from worms exposed to three different attractive odors - benzaldehyde (BA), citronellol (CI) and isoamyl alcohol (IA) - transfers the same odor-specific heritable behavioral changes as early odor stimulations do. This single tRNA molecule could thus carry different odor-specific codes, according to the odors worms were exposed.

The nucleotides of all forms of coding and non-coding RNAs can be chemically modified. To date, more than 160 different chemical modifications of RNA bases have been described. Each modification involves specific reactions catalyzed by enzymes called « writers » (Sarin and Leidel, 2014; Schaefer et al., 2017; Jonkhout et al., 2017; Boccaletto P. et al., 2018).

It is largely admitted that, compared to all other forms of RNAs, tRNAs are the most extensively modified. The combination of modified bases would potentially produce a significant number of chemical variants for a single tRNA molecule.

We hypothesized that specific base modification patterns shape the odor-codes written on $\mathrm{tRNA}^{\text {Ala }}$ (UGC) molecules upon odor-stimulation. We, therefore, analyzed the behavioral effects of mutations inactivating the Elongator complex, ELPC, the only known tRNA bases modifier in C. elegans. Inactivation of the elongator complex sub-units $3(e l p c-3)$ or 1 (elpc-1) impairs differently the chemo-attractive behavior.

elpc-3 deleted worms are no longer attracted to CI, BA or IA. Chemo-attraction is, however, fully rescued to wild-type levels after feeding elpc-3 mutants on naive wild-type tRNA ${ }^{\text {Ala }}$ (UGC). This suggests $e l p c-3$ is required for the synthesis of the chemical form of tRNA Ala (UGC) which supports the development of chemo-attractive responses.

Furthermore, the absence of a functional ELPC-1 in the elpc-1 deleted mutants reverses the outcome of odor-exposure: as opposed to wild-type, early exposure stably decreases adult odor-specific responses of elpc-1 mutants and their progeny. We observed that such negative odor-specific imprinting can be impaired upon addition of odor-specific amounts of the modified base 5-carbamoylmethyl Uridine ( $\mathrm{ncm} 5 \mathrm{U})$ in worm food. Epitranscriptomic regulation of tRNA ${ }^{\text {Ala }}$ (UGC) appears to be an essential molecular mechanism linking environmental inputs and stably inherited changes of the C. elegans chemoattractive behavior.

\section{RESULTS}

Olfactory imprinting is a long-term inherited behavioral change induced by the early olfactory environment. We hypothesized RNAs could convey the olfactory imprints. First, olfactory imprinting was induced, as previously described (Remy and Hobert, 2005), by exposing animals to two different olfactory cues, citronellol (CI) or benzaldehyde (BA) (Figure 1A, odor-exposure). The induction efficiency was tested using the chemotaxis assay described in section "MATERIALS AND METHODS" and in Supplementary Figure Method 1.

Second, total RNA was extracted from CI-exposed, BAexposed or water-exposed control worms. Third, these RNAs were fed to naive larvae (Figure 1A, RNA-feeding). Fourth, once RNA-fed larvae reached adulthood, they were subjected to the chemotaxis assays. Naive worms fed on RNA from CI or BAexposed worms migrate significantly faster toward CI or BA, as if they had been themselves odor-exposed (Figure 1B).

This observation shows that worms exposed to odors during the L1 larval stage produce RNA populations able to alter chemosensory responses of naive animals via ingestion.

To identify which RNA molecules do transfer odor imprints, we separated the large from the small RNAs. We fed naive worms on either the "large RNAs fraction" or the "small RNAs fraction" and observed that only RNAs smaller than 200 nucleotides (nt) can trigger the imprinting (Supplementary Figure 1a). Migration on a $3.5 \%$ agarose gel separates small RNAs into five fractions (A to E bands, insert on Supplementary Figure 1b). After RNA-feeding, we observed that olfactory imprints were exclusively transmitted by the "D" small RNA fraction. Based on co-migration with a double-stranded RNA ladder (L), the " $\mathrm{D}$ " RNA population migrates with an apparent mean size of $45 \mathrm{nt}$ on this non-denaturing electrophoresis condition.

Further fractionation of the naive (NA) and of the imprinting (CI) fractions on denaturing $7 \mathrm{M}$ urea-15\% polyacrylamide gel (Figure 2A) revealed they are composed of several RNA species we named fractions 1 to 8 . We noticed that fractions 2 to 7 represent a typical profile of tRNAs (Figure 2A, left panel). Performing northern-blot analyses using two C. elegans specific probes for tRNA ${ }^{\text {Leu }}$ (AGG) and tRNA ${ }^{\text {Gly }}$ (UCC), we confirmed that fractions 2 to 7 indeed contain the C. elegans tRNA population (Figure $2 \mathbf{A}$, right panel).

We next wanted to know if the imprinting activity is spread over the whole tRNA co-purified population or linked to specific fractions. We cut and eluted each of the indicated 1 to 8 fractions from the polyacrylamide gels. We reconstituted the whole imprinting fraction by mixing fractions 1 to 8 , and the whole tRNA co-migrating RNA populations by combining fractions 2 to 7. Both 1 to 8 (CI Mix 1-8) and 2 to 7 (CI Mix 2-7) mixes extracted from CI-exposed worms, transferred a CIspecific imprint to naive worms, while the corresponding NA mixes (NA Mix 1-8 and NA Mix 2-7) did not (Figure 2B).

After a detailed fraction by fraction analysis, we found that feeding naive with fractions 5 and 6 significantly enhance CI chemo-attraction (Figure 2C).

The C. elegans genome is predicted to encode 596 functional tRNA genes (Duret, 2000; Robertson and Thomas, 2006; Chan and Lowe, 2009).

The tRNAs need to be heavily modified post-transcriptionally to be fully active (Agris, 2015; Duechler et al., 2016). Indeed, the high abundance of tRNA post-transcriptional modifications blocks the progression of reverse-transcriptases, thus cDNA synthesis, making quantitative tRNA sequencing very challenging. Alternative methods have been proposed in the recent literature to obviate these limitations (Pang et al., 2014; Zheng et al., 2015; Cozen et al., 2015; Shigematsu et al., 2017), 
A
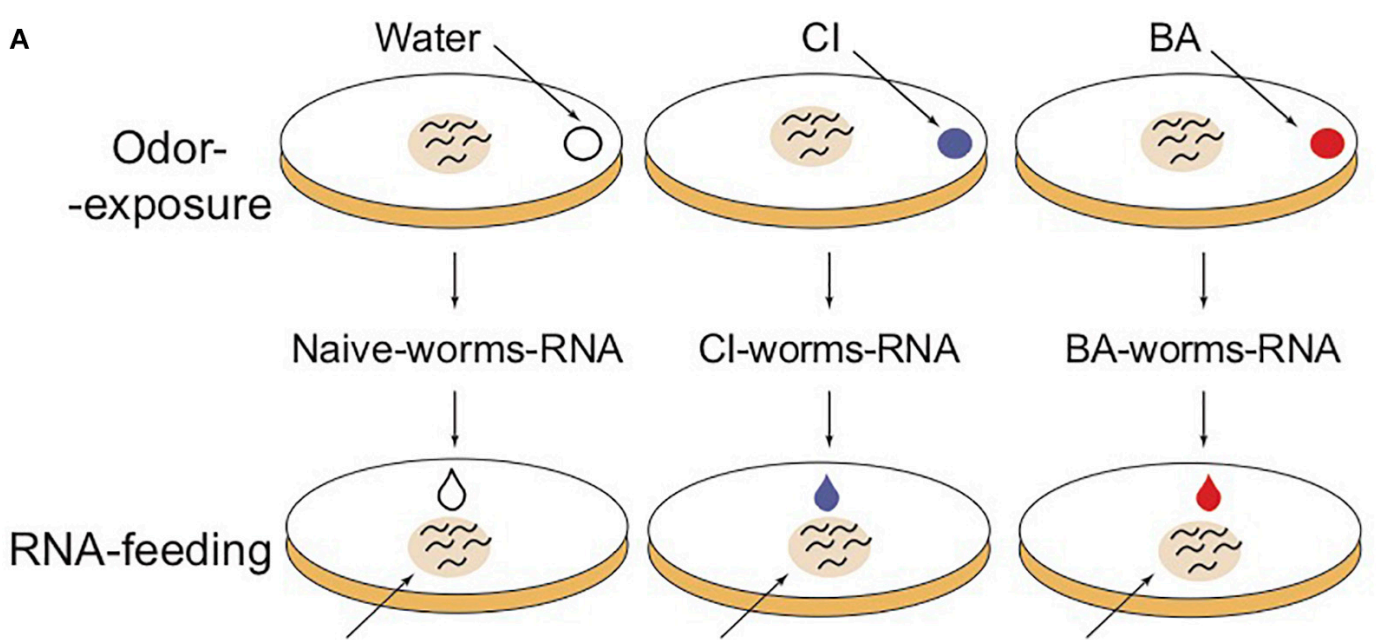

Naive-RNA on naive worms
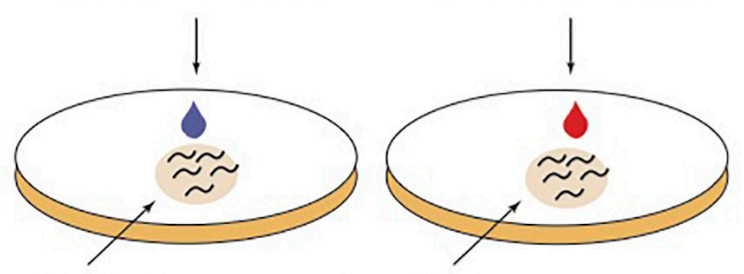

Cl-RNA on

BA-RNA on

naive worms

naive worms

B

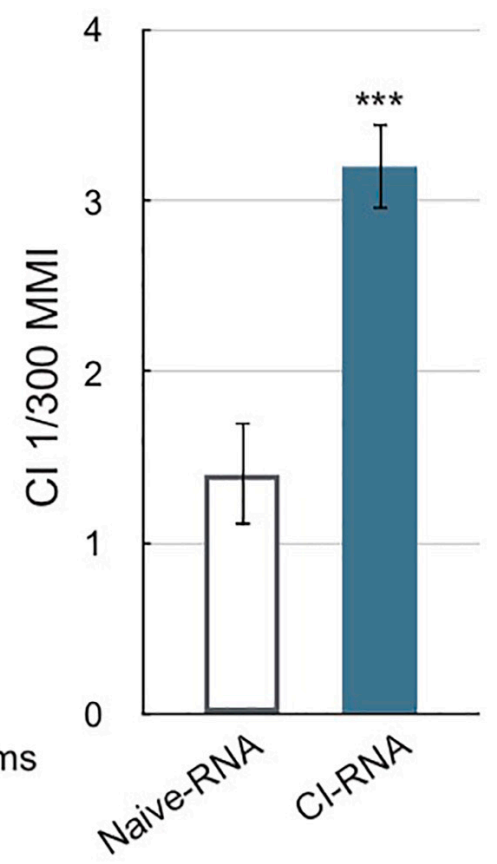

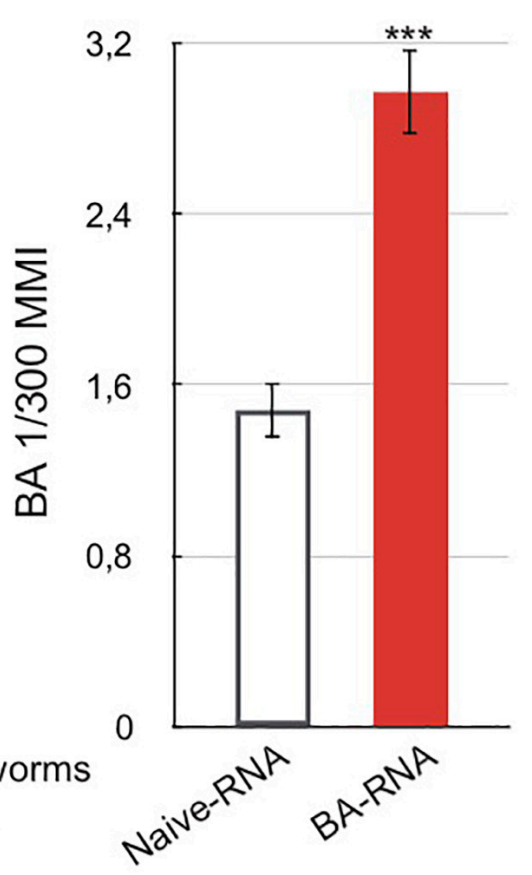

Naive worms fed on:

FIGURE 1 | RNA extracted from odor-exposed worms transfer olfactory imprinting to naive unexposed worms via feeding. (A) Schematic depiction of worms odor-exposure and RNA-feeding. Upper part: a drop of $4 \mu \mathrm{l}$ of citronellol (Cl) or benzaldehyde (BA) or water (for control unexposed) is placed on the lid of each culture plate. Worms produce long-lasting odor-specific imprints when exposed to odors for $12 \mathrm{~h}$ post-hatch at $20^{\circ} \mathrm{C}$, the critical period for olfactory imprinting (Remy and Hobert, 2005). Worms exposed to odors during this period are collected at the adult stage. RNA is extracted from each collected population. Bottom part: a $10 \mu \mathrm{l}$ drop of purified RNA is placed on worm food (E. coli OP50) to be ingested by naive larvae. (B) Chemotaxis assays performed on naive adults fed on different RNA populations. Worms fed on RNA from Cl-exposed (Cl-RNA, blue column) and worms fed on RNA from BA-exposed (BA-RNA, red column) migrate faster toward a Cl source and a BA source, respectively, compared to worms fed on RNA from naive animals (Naive-RNA, white columns). Mean Migration Index (MMI) was determined as described in Supplementary Material (experimental repeats $>4,{ }^{\star \star \star} p$-value $<0.001$ ). 

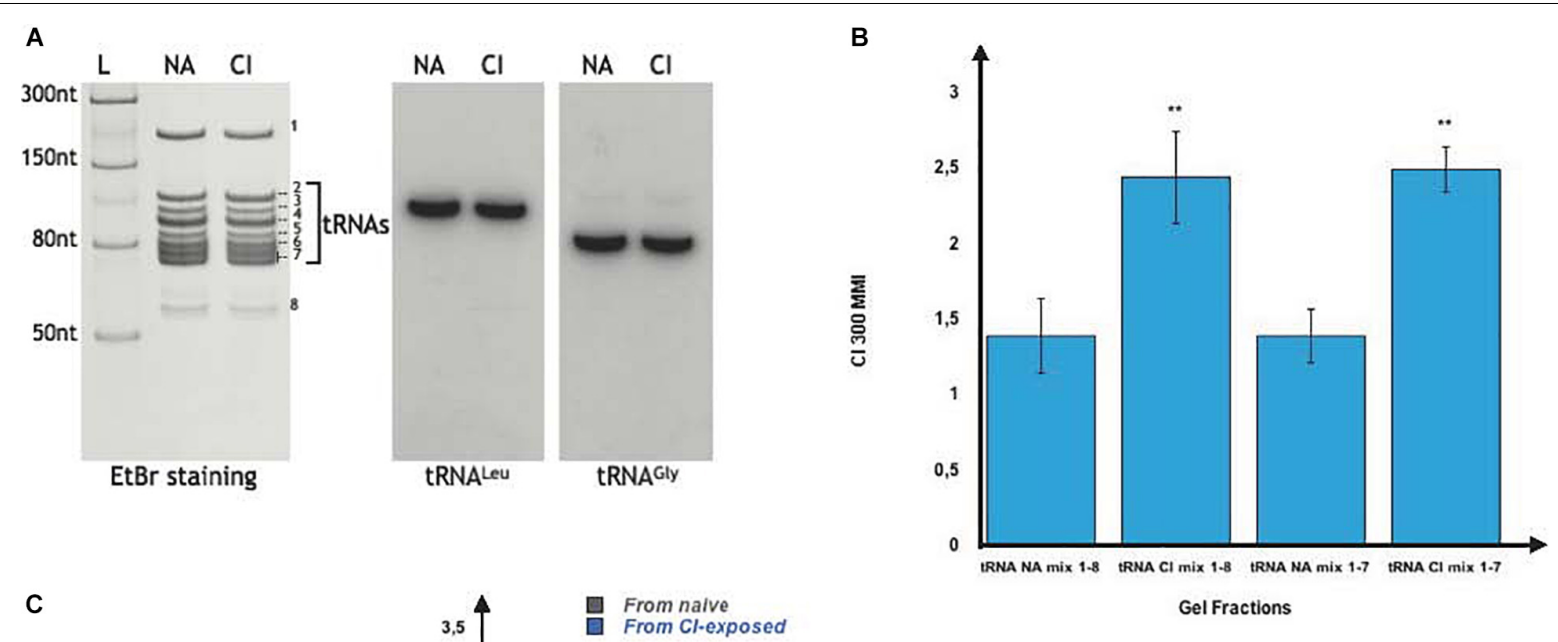

C

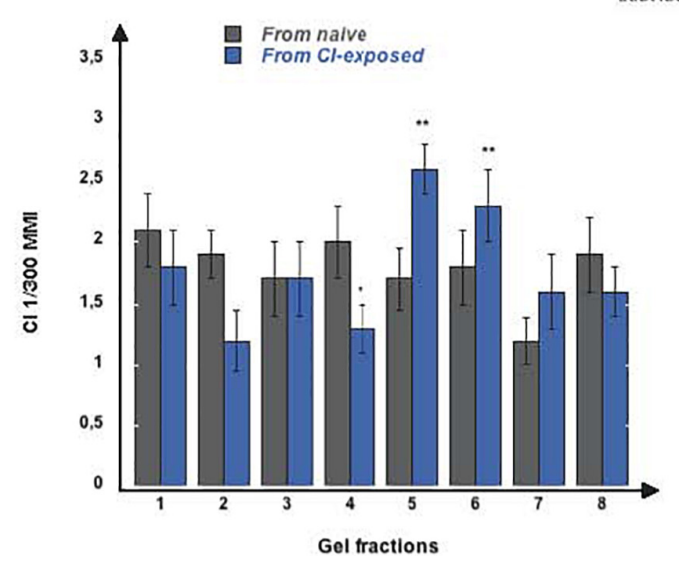

FIGURE 2 I Olfactory imprinting is triggered by the transfer RNAs (tRNAs) containing fraction. (A) Left part: Total small RNA from naive (NA) and from Cl-exposed (Cl) were fractionated on a $7 \mathrm{M}$ Urea 15\% polyacrylamide gel and stained with ethidium bromide (EtBr). Band 1 is the $5 \mathrm{~S}$ rRNA, bands 2 to 7 are tRNAs, while band 8 is made of unknown RNAs. The L well contains the NEB single stranded RNA ladder. Right part: Northern blot analyses were performed on NA and Cl RNA blots with two radiolabeled probes specific to the $C$. elegans tRNA $A_{\text {Leu }}(A A G)$ and tRNA $A_{\text {Gly }}(\mathrm{UCC})$. (B) After elution from the polyacrylamide gels, fractions 1 to 8 or fractions 2 to 7 from naive (NA Mix 1-8 and NA Mix 2-7) and from Cl-exposed (Cl Mix 1-8 and Cl Mix 2-7) were pooled. Naive worms were fed on each pool and assayed for $\mathrm{Cl}$ response. Both of the pooled $\mathrm{Cl}$ mixes trigger a faster migration (MMI) in $\mathrm{Cl}$ gradients than the corresponding NA mixes (experimental repeats $>4,{ }^{* *} p=0.006$; ${ }^{* \star *} p$ < 0.001). (C) Naive N2 were fed on each of the 1 to 8 individual fractions from naive and from Cl-exposed. Only feeding on the tRNA fractions 5 and 6 from $\mathrm{Cl}$-exposed enhances migration in $\mathrm{Cl}$ gradients (MMI), compared to all other tRNA fractions (experimental repeats $>4,{ }^{\star \star \star *} p<0.001$ ).

however none of them is readily available. For this reason, instead of a sequencing strategy, we used biochemical methods to identify which RNAs molecules are able to transfer olfactory imprinting among these tRNA co-purified fractions.

To assess if tRNAs are responsible for imprinting, we combined streptavidin microbeads purification with northern blot analysis. According to the genomic tRNA database (GtRNAdb) predictions, C. elegans uses 46 different anticodons to decode the 20 amino acids. Five anticodons are used for Ser, Arg and Leu, three for Ala, Gly, Pro, Thr and Val, two for Lys, Glu, Gln and Ile, and one for Phe, Asp, His, Met, Tyr, Cys and Trp.

As mentioned above, imprinting is transferred only by fractions 5 and 6 .

tRNAs bearing different anticodons decoding the same amino acid, named tRNA isoacceptors, display a high degree of sequence homology. Since the denaturing gel shown in Figure 2A separates tRNA molecules based not only of their length but also of their nucleotide sequences, we hypothesized that each fraction, including the imprinting fractions 5 and 6, may contain a limited number of isoacceptor tRNAs.
We used a set of $37 \mathrm{nt}$ long oligonucleotides specific to each tRNA isoacceptors family and complementary to their respective tRNAs $3^{\prime}$ halves, for purifications on microbeads, as described in Methods. Most anticodon-specific tRNAs used by C. elegans are represented in our set of tDNA probes, except two out of the five decoding Ser and Arg and one out of the five decoding Leu. For northern blots analysis, we used short aminoacid-specific isodecoder tRNA probes (see section "MATERIALS AND METHODS").

We reasoned that if a mixture of tRNAs eluted from a pool of tDNA oligonucleotides bound to microbeads transfers imprinting, this population should contain the imprinting RNAs.

tRNAs were purified from odor-exposed (CI or BA) worms using 14 different pools made of oligonucleotides specific to 7 to 11 different tRNAs ( $\boldsymbol{A}$ to $\boldsymbol{N}$, Table 1). The pools were designed such as each isotype (amino-acid decoding) tRNA is present in three different pools. Out of the 14 pools, only tRNAs purified from the A, J and $\mathrm{N}$ pools could transfer the imprinting (Table 1).

Pool A is made of oligonucleotides specific to Ala, Arg, Asn, Asp and Cys tRNAs. 
Arg, Asn, Asp and Cys oligonucleotides did not allow the purification of the imprinting tRNAs, as they are also part of the imprinting negative pools $\mathrm{F}$ and $\mathrm{K}$. Pool $\mathrm{J}$ is made of the Tyr, Val, Ala and Arg probes, but Tyr and Val probes are part of the imprinting negative pool $\mathrm{E}$.

TABLE 1 | Alanine tRNAs from odor-exposed worms transfer imprinting to naive.

\begin{tabular}{|c|c|c|c|c|}
\hline \multicolumn{2}{|l|}{ Pooled tDNA Probes } & \multirow{2}{*}{$\begin{array}{c}\begin{array}{c}\text { RNA from } \\
\text { odor-exposed } \\
\text { worms }\end{array} \\
\begin{array}{c}\text { Small RNAs or } \\
\text { Gel-eluted } \\
\text { fractions } 5 \text { and } 6\end{array}\end{array}$} & \multirow{2}{*}{$\begin{array}{c}\begin{array}{c}\text { Behavior after } \\
\text { feeding } \\
\text { eluates }\end{array} \\
\text { Imprinting }\end{array}$} & \multirow{2}{*}{$\begin{array}{l}\text { tRNA isotypes } \\
\text { Ala, Arg, Asn, } \\
\text { Asp, Cys }\end{array}$} \\
\hline $\begin{array}{l}\text { Ala } 1+2+3 \\
\text { Arg } 1+2+3 \\
\text { Asn, Asp, Cys }\end{array}$ & A & & & \\
\hline $\begin{array}{l}\text { Gln } 1+2, \text { Glu } 1+2 \\
\text { Gly } 1+2, \text { His } \\
\text { lle } 1+2\end{array}$ & B & $\begin{array}{l}\text { Small RNAs or } \\
\text { Gel-eluted } \\
\text { fractions } 5 \text { and } 6\end{array}$ & Naive & $\begin{array}{l}\text { Gln, Glu, Gly, } \\
\text { His, lle }\end{array}$ \\
\hline $\begin{array}{l}\text { Leu } 1+2+3+4 \\
\text { Lys } 1+2, \text { Met } 1+2\end{array}$ & C & $\begin{array}{l}\text { Small RNAs or } \\
\text { Gel-eluted } \\
\text { fractions } 5 \text { and } 6\end{array}$ & Naive & Leu, Lys, Met \\
\hline $\begin{array}{l}\text { Phe, Pro } 1+2+3 \\
\text { Ser } 1+2+3\end{array}$ & D & $\begin{array}{l}\text { Small RNAs or } \\
\text { Gel-eluted } \\
\text { fractions } 5 \text { and } 6\end{array}$ & Naive & Phe, Pro, Ser \\
\hline $\begin{array}{l}\text { Thr } 1+2+3, \text { Trp } \\
\text { Tyr, Val } 1+2+3\end{array}$ & $E$ & $\begin{array}{l}\text { Small RNAs or } \\
\text { Gel-eluted } \\
\text { fractions } 5 \text { and } 6\end{array}$ & Naive & Thr, Trp, Tyr Val \\
\hline $\begin{array}{l}\text { Arg } 1+2+3 \\
\text { Asn, Asp, Cys } \\
\text { Gln } 1+2, \text { Glu } 1+2\end{array}$ & $\mathbf{F}$ & $\begin{array}{l}\text { Small RNAs or } \\
\text { Gel-eluted } \\
\text { fractions } 5 \text { and } 6\end{array}$ & Naive & $\begin{array}{l}\text { Arg, Asn, Asp } \\
\text { Cys, Gln, Glu }\end{array}$ \\
\hline $\begin{array}{l}\text { Gly } 1+2 \text {, His } \\
\text { Ile } 1+2 \\
\text { Leu } 1+2+3+4\end{array}$ & G & $\begin{array}{l}\text { Small RNAs or } \\
\text { Gel-eluted } \\
\text { fractions } 5 \text { and } 6\end{array}$ & Naive & $\begin{array}{l}\text { Gly, His, lle } \\
\text { Leu }\end{array}$ \\
\hline $\begin{array}{l}\text { Lys } 1+2 \\
\text { Met } 1+2 \\
\text { Phe, Pro } 1+2+3\end{array}$ & $\mathbf{H}$ & $\begin{array}{l}\text { Small RNAs or } \\
\text { Gel-eluted } \\
\text { fractions } 5 \text { and } 6\end{array}$ & Naive & $\begin{array}{l}\text { Lys, Met, Phe } \\
\text { Pro }\end{array}$ \\
\hline $\begin{array}{l}\text { Ser } 1+2+3 \\
\text { Thr } 1+2+3, \operatorname{Trp}\end{array}$ & 1 & $\begin{array}{l}\text { Small RNAs or } \\
\text { Gel-eluted } \\
\text { fractions } 5 \text { and } 6\end{array}$ & Naive & Ser, Thr, Trp \\
\hline $\begin{array}{l}\text { Tyr, Val } 1+2+3 \\
\text { Ala } 1+2+3 \\
\text { Arg } 1+2+3\end{array}$ & $\mathbf{J}$ & $\begin{array}{l}\text { Small RNAs or } \\
\text { Gel-eluted } \\
\text { fractions } 5 \text { and } 6\end{array}$ & Imprinting & $\begin{array}{c}\text { Tyr, Val, Ala } \\
\text { Arg }\end{array}$ \\
\hline $\begin{array}{l}\text { Asn, Asp, Cys } \\
\text { Glln } 1+2 \text {, Glu } 1+2 \\
\text { Gly } 1+2 \text {, His }\end{array}$ & $\mathbf{K}$ & $\begin{array}{l}\text { Small RNAs or } \\
\text { Gel-eluted } \\
\text { fractions } 5 \text { and } 6\end{array}$ & Naive & $\begin{array}{c}\text { Asn, Asp, Cys } \\
\text { Gln, Glu, Gly, } \\
\text { His }\end{array}$ \\
\hline $\begin{array}{l}\text { Ile } 1+2 \\
\text { Leu } 1+2+3+4 \\
\text { Lys } 1+2\end{array}$ & $\mathbf{L}$ & $\begin{array}{l}\text { Small RNAs or } \\
\text { Gel-eluted } \\
\text { fractions } 5 \text { and } 6\end{array}$ & Naive & Ile, Leu, Lys \\
\hline $\begin{array}{l}\text { Met } 1+2 \\
\text { Phe, Pro } 1+2+3 \\
\text { Ser } 1+2+3\end{array}$ & M & $\begin{array}{l}\text { Small RNAs or } \\
\text { Gel-eluted } \\
\text { fractions } 5 \text { and } 6\end{array}$ & Naive & $\begin{array}{l}\text { Met, Phe, Pro } \\
\text { Ser }\end{array}$ \\
\hline $\begin{array}{l}\text { Thr } 1+2+3, \operatorname{Trp} \\
\text { Tyr, Val } 1+2+3 \\
\text { Ala } 1+2+3\end{array}$ & $\mathbf{N}$ & $\begin{array}{l}\text { Small RNAs or } \\
\text { Gel-eluted } \\
\text { fractions } 5 \text { and } 6\end{array}$ & Imprinting & $\begin{array}{l}\text { Thr, Trp, Tyr } \\
\text { Val, Ala }\end{array}$ \\
\hline
\end{tabular}

Pool $\mathrm{N}$ is made of the Thr, Trp, Tyr, Val and Ala probes, but Thr, Trp, Tyr and Val probes are part of the imprinting negative pools $\mathrm{E}$ and I.

Altogether, we concluded that only the Ala oligonucleotides are present in the three imprinting positive pools $\mathrm{A}$, J and $\mathrm{N}$.

We further proceeded with tRNA purification by isotypespecific probes and confirmed that only the tRNAs eluted from Alanine tDNA probes imprint naive worms, as shown in Figure 3A. It remains possible that other RNA species that co-elute from the Alanine tDNA probes are active species.

Northern blot analyses were performed to identify which tRNAs are present in the imprinting positive fractions (Figure 3B). We show here the analysis of naive (N3 to N6), CI-exposed (C3 to C6) and BA-exposed (B4 to B7) fractions using probes specific to most tRNA isotypes used in Figure 3A. While tRNA ${ }^{\text {Leu }}$ co-migrate with fractions 3 , tRNA ${ }^{\text {Glu }}$ co-migrate with tRNA ${ }^{\text {Gln }}$ mostly in fractions 7 , but are also present in fractions 6 , tRNA ${ }^{\text {Lys }}$ migrate mostly in fractions 6 but are also present in fractions 5. Northern blots support the results obtained using microbeads purification: Alanine tRNAs are primarily present in the imprinting fractions 5 and 6 .

Oligonucleotides 1 to 4, described in Methods, allow the purification of the three different tRNA ${ }^{\text {Ala }}$ isoacceptors used by C. elegans, i.e., tRNA ${ }^{\mathrm{Ala}}$ (AGC), tRNA ${ }^{\mathrm{Ala}}$ (CGC) and tRNA ${ }^{\text {Ala }}$ (UGC). To further discriminate between these Alanine tRNA isoacceptors, each purified tRNA was assayed for its imprinting ability.

RNA species eluted from the microbeads bearing the tRNA ${ }^{\text {Ala }}$ (AGC) or the tRNA ${ }^{\text {Ala }}$ (CGC) probes do not transfer imprinting. By contrast, the RNA species bound to the two probes corresponding to, respectively, the $5^{\prime}$ and the $3^{\prime}$ halves of the tRNA ${ }^{\text {Ala }}$ (UGC) transfer imprinting to naive worms (Figure 3C).

Our findings suggest that the tRNA ${ }^{\mathrm{Ala}}$ (UGC) isodecoder is the only tRNA able to transfer odor-specific imprinting to naive. To further prove this single tRNA molecule is indeed able to carry and transfer several different odor-specific information, we also exposed, besides to BA and to CI, worms to isoamyl alcohol (IA), a third attractive odorant (Bargmann et al., 1993), for which imprinting after early exposure has been demonstrated (Remy and Hobert, 2005).

The use of the microMACS Streptavidin MicroBeads protocol allowed a fast screening of tRNAs activity. We followed the recommended protocol using stringent hybridization conditions and a high salt washing buffer. However, to obtain highly purified tRNA ${ }^{\text {Ala }}$ (UGC) molecules, visualize the purified tRNA, estimate the purification yields and assess the efficiency of imprinting transfer, we scaled up our purification by using chromatography on Streptavidin Sepharose beads coupled to the 3'-biotinylated Ala (TGC)-3' oligonucleotide, as described in section "MATERIALS AND METHODS."

The amount of eluted tRNA was too low to be visible by ethidium bromide staining after gel migration when starting with $1.10^{4}$ worms. Therefore, we used Cy3 labeling, as described in the section "MATERIALS AND METHODS," to detect the tRNAs after gel fractionation.

As shown in Figure 4A, a major band of tRNA ${ }^{\text {Ala }}$ (UGC) from naive, CI, IA or BA-exposed worms was eluted from the beads. 


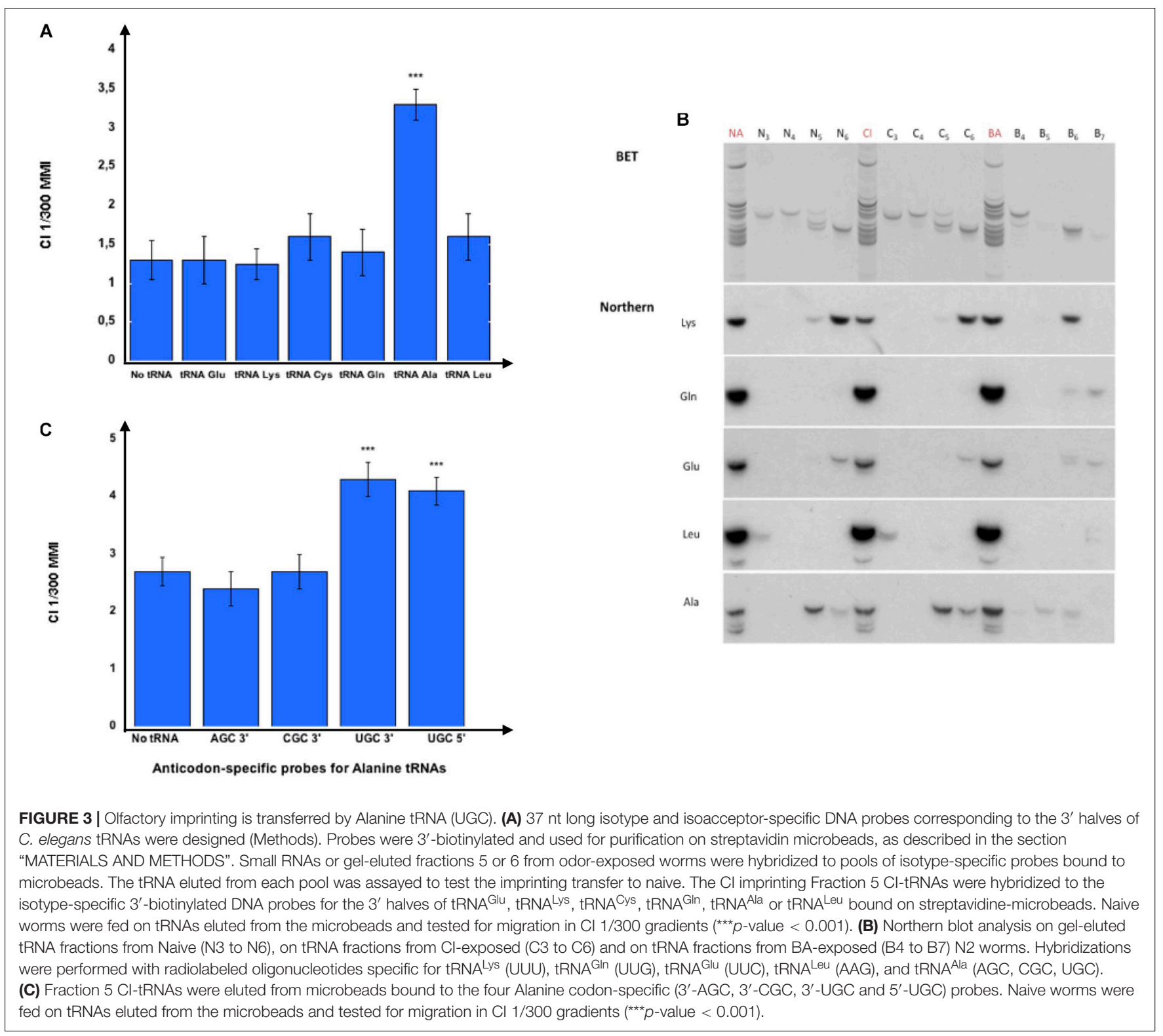

To eliminate any other RNA molecule that may have been copurified, we cut the bands containing the tRNAs out of the gel, and eluted them. The estimated yields were approximately $1 \mathrm{ng}$ of pure tRNA ${ }^{\text {Ala }}$ (UGC) obtained from $1.10^{4}$ adult worms. Naive wild-type worms were fed on 1/10 $\mu$ l of each gel-eluted tRNAs and assayed for CI, IA or BA chemotaxis. Feeding naive worms on gel-eluted tRNA ${ }^{\text {Ala }}$ (UGC) from CI-exposed (CI) worms increases chemo-attraction to $\mathrm{CI}$, compared to feeding tRNA ${ }^{\mathrm{Ala}}$ (UGC) from naive unexposed (NA) worms. Feeding on tRNA ${ }^{\text {Ala }}$ (UGC) from IA or BA-exposed (IA, BA) worms, respectively increases IA or BA responses specifically, compared to feeding tRNA $^{\mathrm{Ala}}$ (UGC) from naive (NA) animals (Figure 4B).

These results strongly suggest that odor-stimulated worms produce odor-specific forms of a unique tRNA ${ }^{\text {Ala }}$ (UGC) molecule, each carrying and transferring odor-specific information, according to a specific early olfactory experience.
Furthermore, imprinting is efficiently transferred by very low amounts of tRNA; we found that dilutions containing $0.001 \mu \mathrm{l}$ of each gel-eluted tRNAs bands (shown on the Figure 4A gel), with less than $1 \mathrm{pg}$ of tRNA ${ }^{\mathrm{Ala}}$ (UGC), transfer imprinting to a population of 20 worms.

The F1 unexposed worm generation inherited the parental enhanced response after odor-exposure, while the F2 generation lost it. However, olfactory imprinting is stably fixed and stably inherited in worm populations after five worm generations were odor-exposed (Remy, 2010).

Imprinting via tRNA ${ }^{\text {Ala }}$ (UGC) feeding might be able to recapitulate the inheritance pattern of imprinting after odorexposure. As schematically outlined (Figure 5), we compared CI responses of the seven CI-tRNA fed generations and their naive progeny grown without tRNA addition until the fourth generation. We found that a CI imprint is passed to the 


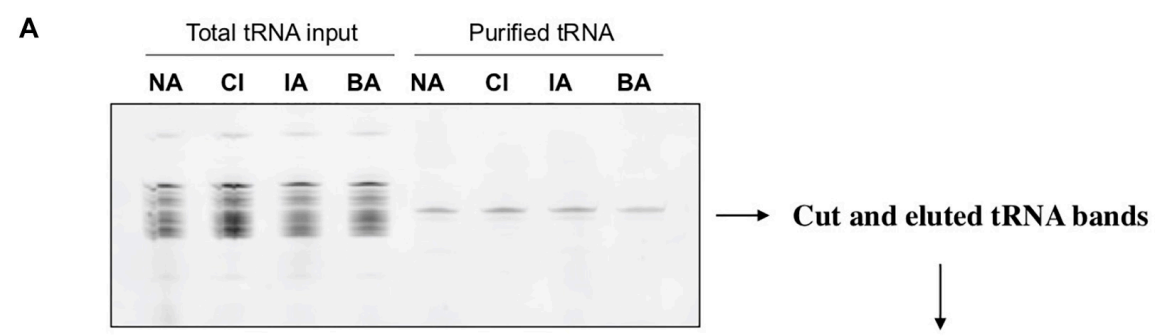

Feeding naive worms

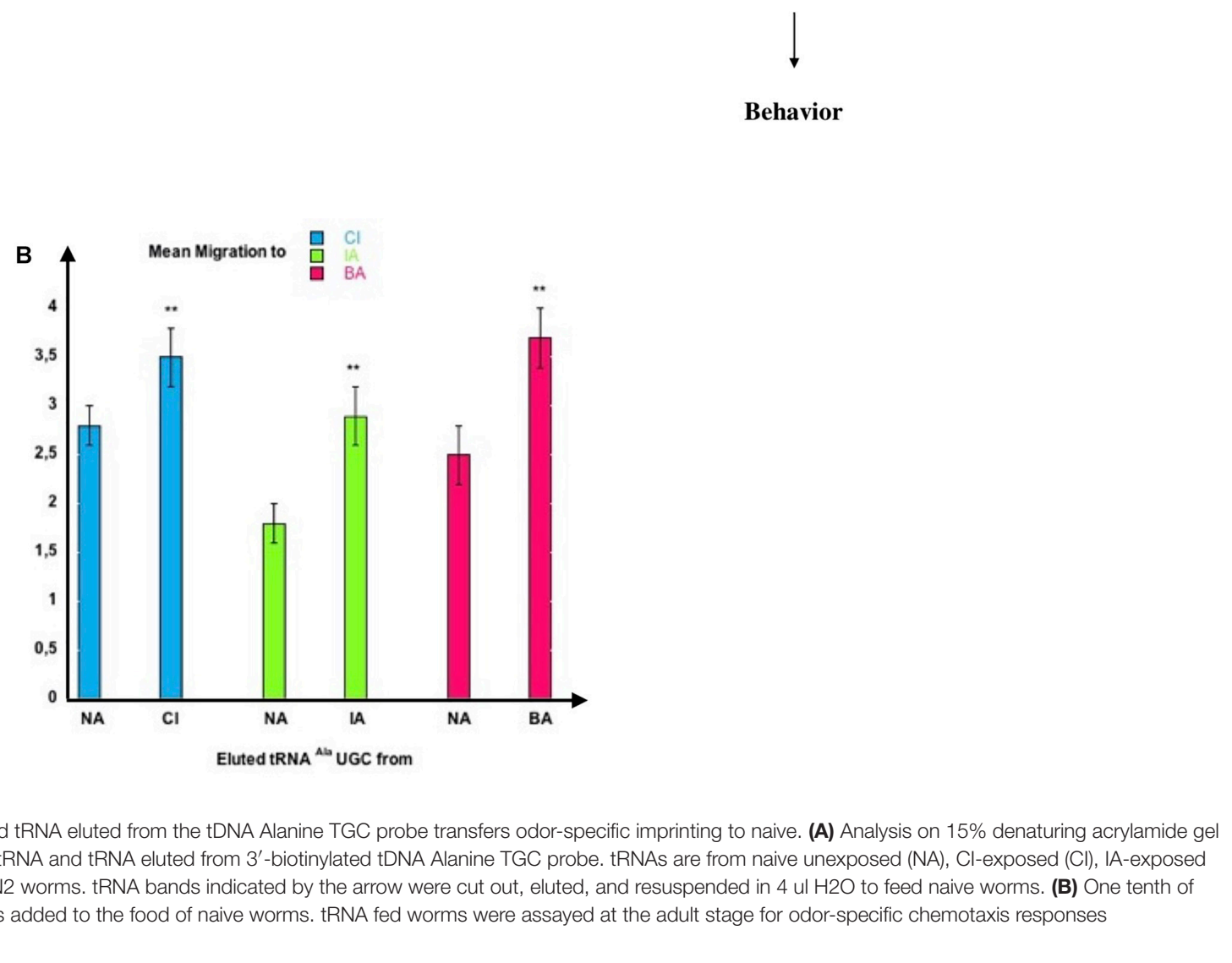

FIGURE 4 | Highly purified tRNA eluted from the tDNA Alanine TGC probe transfers odor-specific imprinting to naive. (A) Analysis on 15\% denaturing acrylamide gel of pCp-Cy3 labeled total tRNA and tRNA eluted from 3'-biotinylated tDNA Alanine TGC probe. tRNAs are from naive unexposed (NA), Cl-exposed (CI), IA-exposed (IA) or BA-exposed (BA) N2 worms. tRNA bands indicated by the arrow were cut out, eluted, and resuspended in $4 \mathrm{ul} \mathrm{H2O}$ to feed naive worms. (B) One tenth of each gel-eluted tRNA was added to the food of naive worms. tRNA fed worms were assayed at the adult stage for odor-specific chemotaxis responses $\left({ }^{\star *} p\right.$-value $\left.<0.01\right)$.

first but not to the second naive generation issued from one to five generations of CI-tRNA fed animals. However, CI imprinting is stably maintained in naive generations issued from worms fed on CI-tRNA at least for six successive generations (Figure 5). To assess the stability of inheritance after the 6th CI-tRNA fed generation, we grew more generations without adding tRNAs. We observed that multi-generationally tRNA-triggered imprinting, as odor-triggered imprinting, is stably maintained in worm progeny. Although it takes six instead of five generations, odor-tRNAs feeding elicit the same long-term stably inherited behavioral change as early odorexposure.

To alter odor responses and imprint next generations, odorspecific tRNAs added to worm food must enter worm tissues through the intestinal cells. The cloverleaf-like tRNA secondary structure is made of loops joined by double-stranded stretches.
Uptake and diffusion of double-stranded RNA (dsRNA) support systemic silencing by RNA interference in C. elegans. We hypothesized tRNAs could take the paths used by dsRNA.

We studied imprinting and its inheritance in mutant worms bearing amino-acid substitutions in one of the two C. elegans dsRNA selective transporters SID-1 or SID-2 (Jose et al., 2009; McEwan et al., 2012). The dsRNA selective transporter SID2 is exclusively localized to the apical membrane of intestinal cells, where it is responsible for the initial binding and internalization of dsRNA from the intestinal lumen (Marré et al., 2016). Due to its high expression in germ-line cells, the dsRNA selective importer SID-1 has been involved in transgenerational diffusion of neuronally expressed mobile dsRNAs (Devanapally et al., 2015).

Worms with sid-1 (qt2), sid-1 (pk3321) and sid-2 (qt13) mutations show a wild-type imprinting behavior after an early 


\title{
$00000 \mathrm{~F} 1+\mathrm{n}$ \\ 000000 F2+n \\ $0000000 \mathrm{~F} 3+n$ \\ $00000000 \mathrm{~F} 4+\mathrm{n}$ \\ $000000000 \mathrm{~F} 5+\mathrm{n}$ \\ $0000000000 \mathrm{~F} 6+n$ \\ $00000000000 \mathrm{F7+n}$ \\ Unstably inherited imprinting \\ Stably inherited imprinting
}

\author{
Generations of N2 wt worms
}

\section{O Feeding tRNA Ala UGC from $\mathrm{Cl}$-exposed, Imprinting behavior}

\author{
No tRNA feeding, Imprinting behavior
}

\section{O No tRNA feeding, Naive behavior}

FIGURE 5 | Olfactory imprinting triggered by tRNA Ala (UGC) can be fixed and stably inherited after feeding at least six generations. Imprinting segregation in the progeny of multigenerational imprinting tRNA fed worm populations (schematic representation). $\mathrm{Cl}$ imprint (Cl-tRNA) produced by $\mathrm{Cl}$-exposed worms was eluted from microbeads bound to the Alanine tDNA $3^{\prime}$-TGC probe. Naive worm populations were fed on Cl-tRNA for one (F1) to seven (F7) successive generations (O). Naive generations grown without addition of RNAs were obtained from each of the seven ( $F 1+n$ to $F 7+n$, here $n=4)$ Cl-tRNAs fed populations. Cl-tRNAs fed worms passed a Cl-imprint to the first naive generation (O). Imprinting is definitely lost at the second naive generation issued from one to five $\mathrm{Cl}$-tRNAs fed generations $(\mathrm{O} \bigcirc \mathrm{O} \ldots$. ); by contrast, it is fixed and definitely maintained in worm populations issued from six and from seven $\mathrm{Cl}$-tRNAs fed generations $(\mathrm{O} O \mathrm{O} \mathrm{O} \ldots$..).

odor exposure (Table 2, Odor-exposure). While odor-triggered imprinting was $\mathrm{F} 1$ inherited in $\mathrm{N} 2$ wild-type and sid-2 (qt13) mutant worms, imprinting inheritance was impaired by the two sid-1 (qt2) and sid-1 (pk3321) substitutions mutations (Table 2, Inheritance). Furthermore, while CI-RNA feeding does imprint naive wild-type, sid-1 (qt2) and sid-1 (pk3321) worms, the sid2 (qt13) mutant worms do not acquire a CI imprint via RNA feeding (Table 2, RNA feeding). These results suggest intertissular and intergenerational imprinting tRNAs use the SID-1 and SID-2 dependent paths described for linear dsRNA motility in C. elegans.

When present in the first C. elegans larval stage environment, attractive odors would trigger the production of different forms of tRNA ${ }^{\text {Ala }}$ (UGC), each bearing an odor-specific signature. The technology available to analyze RNA chemical modifications is making progress and rapidly developing. However, based on biochemical or sequencing approaches, these methods mostly apply to known identified single modifications. To this day, there is no straight forward available method able to describe accurately the whole quantitative and qualitative pattern of bases modifications present on a single purified RNA molecule (Schaefer et al., 2017; Jonkhout et al., 2017; Motorin and Helm, 2019).
tRNAs bases are extensively modified. The combinations of modified nucleotides potentially produce a high number of chemically different tRNA molecules. How such combinatorial chemical diversity is created, controlled and linked to tRNA biological functions remains to be described. We hypothesize those differential combinations of base modifications would form the odor-specific codes carried by $\mathrm{tRNA}^{\mathrm{Ala}}$ (UGC) after odor stimuli.

Aiming to identify some differences between naive and odor-induced tRNAs, we based our investigations within the scope of the currently limited knowledge on tRNA modifications in C. elegans. Most tRNA-modifying enzymes and biosynthesis pathways are described in the yeast but remain unknown in the worm.

Due to the essential role of tRNAs in protein translation, the most studied tRNA chemical modifications have been those affecting the wobble position 34, the first base of the anticodon, in particular Uridine 34.

Wobble $U$ modifications are considered of critical functional importance in codon-anticodon recognition as they might improve tRNA aminoacylation kinetics and prevent translational frame-shifts (Larsen et al., 2015; Nedialkova and Leidel, 2015; Deng et al., 2015). 
TABLE 2 | tRNAs use the SID-1 and SID-2 dsRNA-specific transporters to, respectively, support imprinting inheritance and imprinting transmission via feeding.

\begin{tabular}{lcccc}
\hline $\begin{array}{l}\text { Behavior } \\
\text { Genotypes }\end{array}$ & $\begin{array}{c}\text { Chemotaxis of } \\
\text { naive } \\
\text { unexposed }\end{array}$ & $\begin{array}{c}\text { Imprinting after } \\
\text { odor-exposure }\end{array}$ & $\begin{array}{c}\text { Imprinting } \\
\text { Inheritance }\end{array}$ & $\begin{array}{c}\text { Imprinting after } \\
\text { Cl-RNA feeding }\end{array}$ \\
\hline N2 wt & $1.6 \pm 0.2$ & $2.8 \pm 0.3$ & $2.8 \pm 0.3$ & $2.9 \pm 0.3$ \\
& & $p<0.001$ & $p<0.001$ & $p<0.001$ \\
sid-1 (qt2) & $1.8 \pm 0.3$ & $3.2 \pm 0.2$ & $1.7 \pm 0.3$ & $3.0 \pm 0.2$ \\
& $1.8 \pm 0.3$ & $2.9 \pm 0.2$ & $1.8 \pm 0.3$ & $2.8 \pm 0.3$ \\
sid-1 & & $p<0.001$ & $(=n a i v e)$ & $p<0.001$ \\
(pk3321) & $0.4 \pm 0.2$ & $1.5 \pm 0.2$ & $1.6 \pm 0.2$ & $0.5 \pm 0.2$ \\
sid-2 (qt13) & & $p<0.001$ & $p<0.001$ & $(=n$ naive) \\
& (A) Naive Cl & (B) Cl-exposed & (C) Unexposed & (D) Cl-RNA fed \\
& MMl & Cl MMl & F1 generation & Cl MMl \\
& & & Cl MMl &
\end{tabular}

Wild-type N2 larvae and larvae with the sid-1 (qt2), sid-1 (p/3321) and sid-2 (qt13) mutations were either Cl 1/300 exposed, fed on 100 ng of RNA from Cl 1/300 exposed N2 (Cl-RNA), or unexposed as control naive. Mean Migration Indices (MMI) to $\mathrm{Cl}$ 1/300 of naive worms (A), of $\mathrm{Cl}$-exposed worms $(\mathrm{B})$, of unexposed F1 worms from Cl-exposed (C), and of Cl-RNA fed worms (D), were determined as described (experimental repeats $>4$ ).

tRNA $^{\text {Ala }}$ (UGC) indeed carries uridine at position 34, by contrast to the two other Alanine tRNA (AGC) and tRNA (GCG). Several U34 modifications require the activity of the tRNA modifying Elongator complex (Karlsborn et al., 2014).

The yeast and mammalian Elongator complexes are composed of two copies of two sub-complexes associating the three ELP-12-3 and the three ELP-4-5-6 subunits (Glatt et al., 2012; Dauden et al., 2017). However, the C. elegans elongator complex, ELPC, may function as a 1-2-3 sub-complex, since the elongator subunits 5 and 6 genes are absent from the worm genome.

Recent structural and functional analysis of tRNAs processing by the yeast elongator complex indicates that the carboxymethylation (cm5) of 11 tRNAs carrying U34, including the tRNA ${ }^{\text {Ala }}$ (UGC), requires the activity of the catalytic 1-2-3 sub-complex (Dauden et al., 2019). The $\mathrm{cm} 5$ modification will further lead to the formation of 5-methoxycarbonylmethylated (mcm5), 5-methoxycarbonylmethyl-2-thiolated (mcm5S2) and 5-carbamoylmethylated (ncm5) modified uridines. In the yeast, ELP-1 and ELP-3 sub-units both interact with tRNAs. The Methanocaldococcus infernus archeon, which expresses none but the ELP-3 sub-unit, does perform the U34 carboxymethylation reaction. It has been shown that the C. elegans elongator complex also controls the synthesis of the mcm5, mcm5S2 and ncm5 modifications of tRNA uridines (Chen et al., 2009).

Elongator has been implicated in a great variety of nuclear and cytoplasmic cellular functions, including transcription elongation, chromatin remodeling, exocytosis, zygotic paternal DNA demethylation, and neuronal development (Creppe et al., 2009; Okada et al., 2010; Solinger et al., 2010; Tielens et al., 2016; Dalwadi and Yip, 2018). The multifunctionality of Elongator could be partly explained by structural analysis (Dauden et al., 2017, 2019). ELP3 contains a C-terminal Lysine Acetyl Transferase (KAT) domain and a domain with sequence homology with the S-adenosylmethionine (SAM) domain. EPL-3
KAT could acetylate several targets, including histones and the neuronal alpha-tubulin (Creppe et al., 2009; Solinger et al., 2010). In the mouse, the radical SAM domain but not the KAT domain has been involved in paternal genome demethylation, suggesting the two ELP3 functional domains could play different roles (Okada et al., 2010). Based on over $80 \%$ primary sequence identity, ELPC-3, the worm Elongator sub-unit 3, may carry the same functional domains described for yeast and mammals.

Recent studies reported the effects of mutations inactivating the $C$. elegans Elongator sub-units. It is important to notice that worms harboring mutations in either elpc-1, elpc-2 or elpc3 genes display neuronal and behavioral phenotypes (Chen et al., 2009; Solinger et al., 2010; Kawamura and Maruyama, 2019). This suggests remarkable evolutionary conservation of the elongator functions in neurodevelopment (Creppe et al., 2009; Kojic and Wainwright, 2016).

To see if ELPC-3 could be involved in chemo-attraction regulation, we used worms carrying different chromosomal deletions of the elpc-3 gene (Figure 6). The 1503 bp ok2452 deletion span the whole putative ELPC-3 Radical SAM domain, mapped by homology between amino acids 89 and 300, while the 355 bp tm3120 deletion may leave it intact. We mapped the putative C-terminal ELPC-3 KAT domain between amino acids 436 and 547, based on sequence homology with the yeast and mammalian ELP-3 KAT domains. Using the CRISP-Cas9 technology, we produced worms with a 375 bp deletion, called here del-KAT, which eliminates the KAT domain from ELPC-3 (Figure 6A). We found that, though to variable extents, all tested elpc-3 deletion mutants display significantly reduced chemoattractive responses to CI, BA and IA, compared to wild-type N2 worms (Figure 6B).

We next tested the behavioral effects of the three different elpc1 gene deletions described in Figure 7A. Worms bearing the $n g 10$ 2050 bp deletion could not produce ELPC-1, while worms with the $t m 2149275 \mathrm{bp}$ and the $t m 1168495 \mathrm{bp}$ elpc-1 deletions could produce C-terminal truncated forms of ELPC-1. By contrast to the elpc-3 mutants, chemo-attraction is not significantly affected by the elpc-1 gene deletions, compared to wt N2 (Figure 7B).

We reasoned that if the chemotaxis defects of elpc-3 worms are due to impaired modifications of the tRNA bases, then providing wild-type tRNA through feeding might rescue the behavioral phenotype. We cut and eluted the naive wild-type (NA) tRNAs co-migrating fractions 2 to 7 from the gel shown in Figure 2A.

After feeding elpc-3 (tm3120) worms on the pooled 2 to 7 fractions or on each fraction, separately, we found that the whole population of naive tRNAs (2-7) and the Alanine tRNAs containing fractions 5 and 6 (N5 and N6 on Figure 3) indeed restore CI chemotaxis (Figure 8A). We next asked if feeding the microbead-purified wild-type naive tRNA ${ }^{\text {Ala }}$ (UGC) is enough to restore the chemo-attractive defects of elpc-3 mutants. As shown in Figure 8B, elpc-3 (tm3120) and elpc-3 (2452) worms indeed recover a wild-type behavior after being fed wild-type tRNA $^{\text {Ala }}$ (UGC).

We next demonstrated that none of the other Alanine tRNAs rescue the elpc-3 behavioral defects. As shown in Figure 8C, the tRNA $^{\text {Ala }}$ (UGC), but neither the tRNA ${ }^{\text {Ala }}$ (AGC) nor the tRNA ${ }^{\text {Ala }}$ 


\section{A}

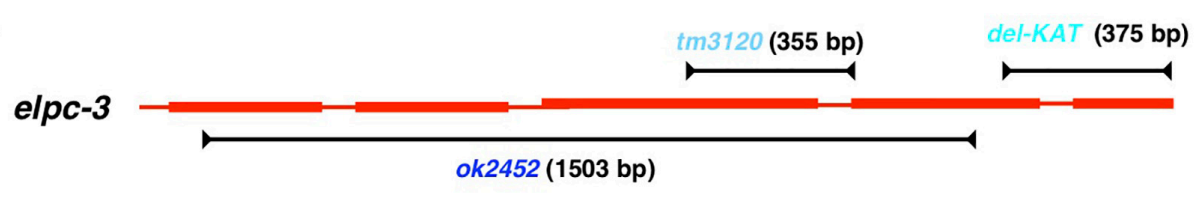

\section{ELPC-3}

Radical SAM domain (aa 89 to 300 ) KAT domain (aa 436 to 547)

\section{B}

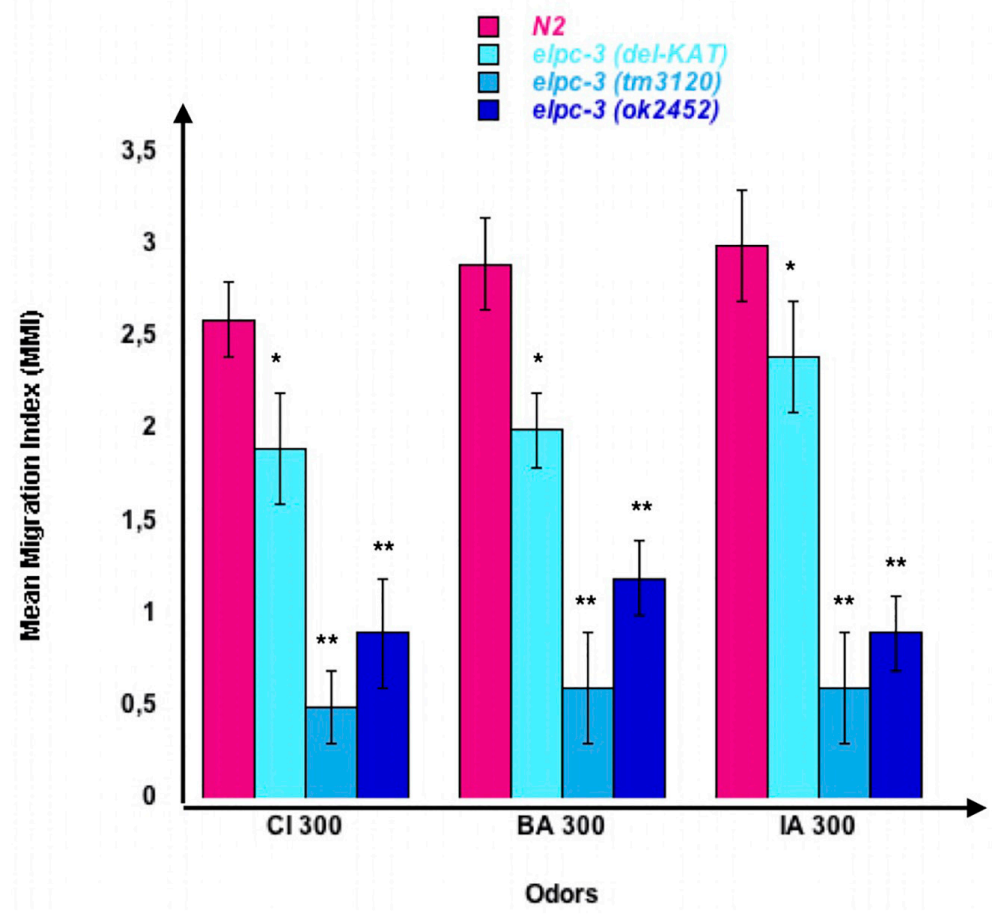

FIGURE 6 | C. elegans chemo-attractive behavior requires a functional elongator complexe sub-unit 3 (ELPC-3). (A) Schematic representation of the C. elegans Elongator sub-unit 3 (e/pc-3) gene, e/pc-3 gene deletion, and of the putative Radical SAM and Lysine Acetyl Transferase KAT domains of the ELPC-3 protein. (B) Chemo-attractive behavior of worms bearing different deletion of the elpc-3 gene, compared to wild-type N2. Mean Migration Indices (MMI) to Citronellol (CI 300), Benzaldehyde (BA 300) and Isoamyl alcohol (IA 300) were determined as described for 4 days old worms (experimental repeats $\geq 4,{ }^{*} p$-value $<0.05$, ${ }^{* *} p$-value $\left.<0.01\right)$.

(CGC) fully rescues the defective behavior of elpc-3 (tm3120) mutants (Figure 8C).

We did not observe significant effects of elpc-1 deletions on chemo-attraction (Figure $7 \mathbf{B}$ ). Moreover, feeding wild-type naive tRNA ${ }^{\text {Ala }}$ (UGC) modifies neither the behavior of elpc1 (tm2149) nor the behavior of elpc-1 (tm11684) mutants (Supplementary Figure 2).

elpc-3 mutants provide with the wild-type tRNA ${ }^{\text {Ala }}$ (UGC) via feeding acquire a wild-type behavior. The rescue of elpc-3 phenotype by wt tRNA ${ }^{\mathrm{Ala}}$ (UGC) suggests elpc-3 worms may produce a "defective " form of tRNA Ala $^{\text {a }}$ (UGC), presumably with an altered chemical composition, unable to support the development of a wild-type chemoattractive behavior.
We then hypothesized that if the development of a wildtype behavior requires the wild-type tRNA ${ }^{\text {Ala }}$ (UGC) chemical composition, then the "defective» RNA $^{\text {Ala }}$ (UGC) produced by elpc-3, could transfer a "defective » elpc-3 behavioral phenotype to wild-type via feeding. Furthermore, the elpc-1 mutants, displaying a wild-type chemo-attraction, would produce the " permissive " wild-type chemical form of tRNA ${ }^{\text {Ala }}$ (UGC), able to rescue the elpc-3 phenotype.

Therefore, we purified tRNA ${ }^{\text {Ala }}$ (UGC) from elpc-3 and from elpc-1 worms to assess their behavioral effects. As shown on Figure 9A, feeding wild-type N2 worms on, respectively, tRNA Ala (UGC) purified from either elpc-3 (tm3120), elpc-3 (ok2452) or elpc-3 (del-KAT) worms, indeed significantly impairs CI 300 and IA 300 attraction. 


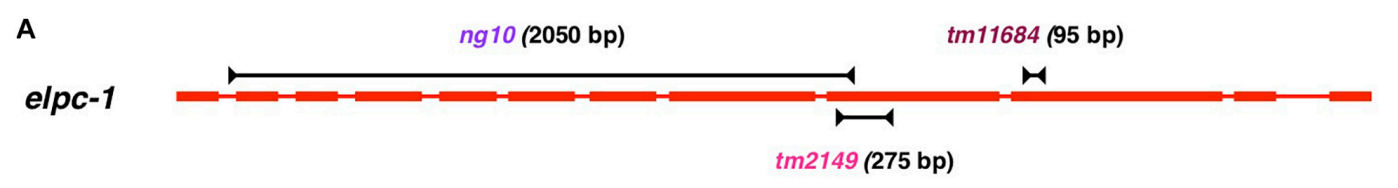

B

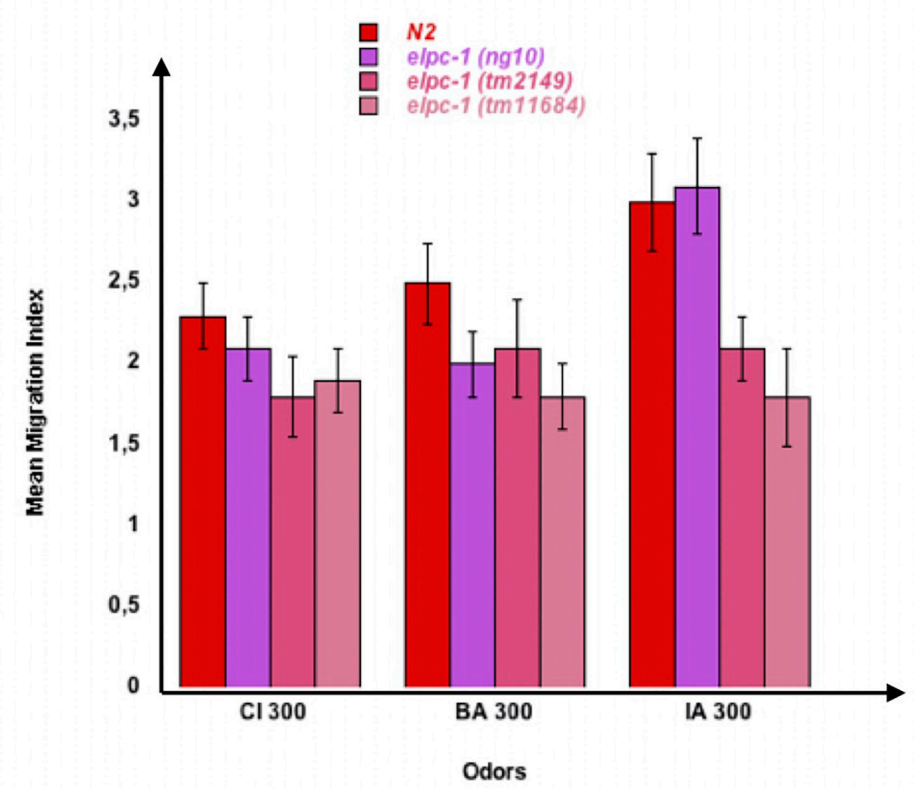

FIGURE 7 | Inactivating the C. elegans elongator complexe sub-unit 1 (ELPC-1) does not significantly modify chemo-attractive responses. (A) Schematic representation of the $C$. elegans Elongator sub-unit 1 gene, and of the elpc-1 gene deletions. (B) Chemo-attractive behavior of worms bearing three different deletion of the elpc-1 gene, compared to wild-type N2. Mean Migration Indices (MMI) to Citronellol (Cl 300), Benzaldehyde (BA 300) and Isoamyl alcohol (IA 300) were determined as described for 4 days old worms (experimental repeats $\geq 4$ ).

Furthermore, feeding tRNA ${ }^{\text {Ala }}$ (UGC) purified from either elpc-1 (tm2149) or elpc-1 (tm11684) worms fully rescues the CI 300 chemo-attractive defect of elpc-3 (ok2452) worms, while feeding its own tRNA ${ }^{\mathrm{Ala}}$ (UGC) or the elpc-3 (tm3120) tRNA ${ }^{\mathrm{Ala}}$ (UGC) has no rescuing effect (Figure 9B).

Importantly, the elpc-3 rescuing effects of wt or elpc-1 tRNA $^{\text {Ala }}$ (UGC) (Figure 8A), or the transfer of an elpc-3 mutant phenotype to wt worms by elpc-3 tRNA ${ }^{\text {Ala }}$ (UGC) (Figures 9A,B) do not last beyond the tRNA fed worm generation.

The elpc-1 deletions do not affect chemo-attraction (Figure 7B). We assessed olfactory imprinting in these mutants. In contrast with wild-type, early odor-exposure or odor-specific tRNA $^{\text {Ala }}$ (UGC) feeding, do not increase but stably decrease odor responses in elpc-1 mutants. CI or BA-exposed elpc-1 (ng10), elpc-1 (tm2149) or elpc-1 (tm11684) worms display a significant reduction of $\mathrm{CI}$ or $\mathrm{BA}$ chemo-attractive responses, compared to naive unexposed (Figure 10A).

Attraction to BA, IA, or CI, significantly decreased after elpc1 (ng10) and elpc-1 (tm2149) mutants were fed on, respectively, highly purified by gel elution (as shown on Figure 4), wild-type BA, IA or CI-tRNA ${ }^{\text {Ala }}$ (UGC) (Figure 10B).
Thus, in the absence of a functional ELPC-1, olfactory imprints have a negative impact on future adult chemoattraction, as opposed to wild-type.

We furthermore demonstrated that worms could be negatively imprinted by one or several odors, as negative imprinting is stably inherited over further generations. As shown in Figure 11, we submitted all three elpc-1 deletion mutants to sequential odorexposures. One generation was exposed to a single odorant, the next generation was (or not) exposed to a second odorant, and the third generation exposed (or not) to a third odorant. IA-exposed worms remain attracted to $\mathrm{BA}$ as naive, while sequentially exposed worms to $\mathrm{CI}$ then to $\mathrm{BA}$ ( $\mathrm{CI} \mathrm{BA}$ ) or to $\mathrm{CI}$ then to $\mathrm{BA}$ then to IA (CI BA IA), lost BA attraction (left part of Figure 11). In the same manner, IA response was lost by IA-exposed and by CI BA IA-exposed elpc-1 worms, while it remained unaffected in CI BA exposed, compared to naive (right part of Figure 11). The inheritance of odor-specific lowered attraction to either a single, two, or three different odorants was assessed up to the tenth generation. Altogether, our data clearly indicate that the elongator complex is essential for the development and the regulation of $C$. elegans chemo-attractive behavior. 

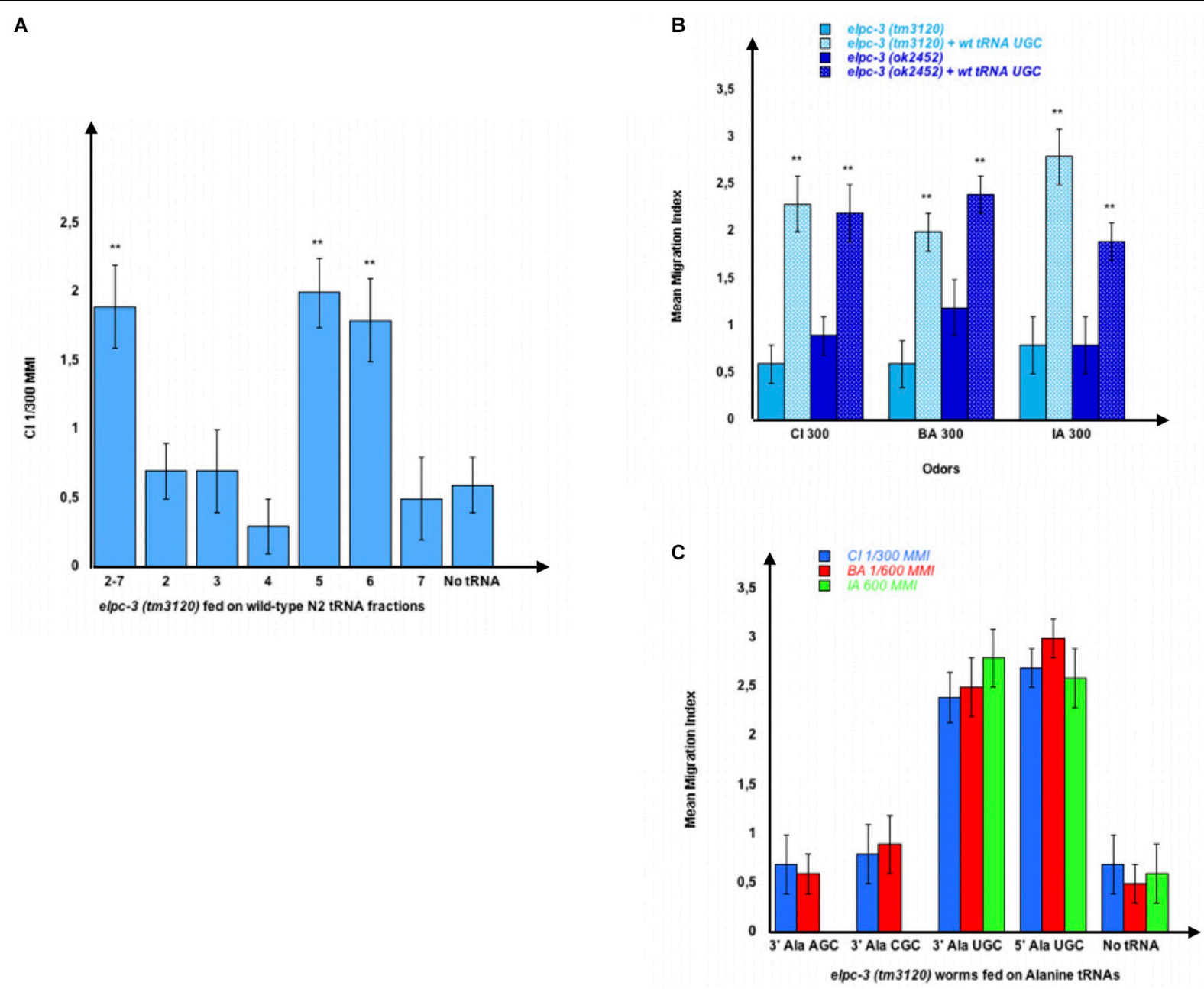

FIGURE 8 | Feeding wild-type tRNA Ala UGC fully restores chemo-attraction in elpc-3 mutants. (A) Mean migration indices of elpc-3 (tm3120) mutants after they were either unfed (No tRNA), fed on the pooled N2 tRNA fractions (2-7) or fed on individual N2 tRNA fractions (1 to 7). tRNA fractions were gel-extracted as shown on Figure 2. (B) Chemo-attractive responses of naive or of wild-type tRNAAla UGC fed elpc-3 (tm3120) and elpc-3 (ok2452) mutant worms. Mean Migration Indices $(\mathrm{MMI})$ in gradients of Citronellol $(\mathrm{Cl})$, Benzaldehyde $(\mathrm{BA})$ and Isoamyl alcohol $(\mathrm{IA})$ at the indicated dilutions, were determined as described (experimental repeats $\geq 4$, ${ }^{* *} p$-value $\left.<0.01\right)$. (C) tRNAAla UGC, but not tRNAAlaAGC nor tRNAAla CGC feeding rescues the chemotaxis defects of elpc-3 (tm3120) worms.

It has been reported that the total tRNA populations extracted from elpc-3 (tm3120) and elpc-1 (2149) lack the mcm5S2U and the ncm5U modifications, and instead contain S2U, as opposed to wild-type tRNAs which carry both mcm5S2U and ncm $5 \mathrm{U}$ but does not contain S2U (Chen et al., 2009). It is believed that these modifications would mainly affect codon-anticodon pairing for the three tRNAs with $U$ at positions 34 and 35, namely Lys (UUU), Glu (UUC) and Gln (UUG).

Even if proven true, this simple picture appears far too simple to account for our observations for several reasons:

- There is no specific information regarding if and how U34 is modified in the eleven U34 containing tRNAs, that includes Ala (UGC);

- These uridine modifications could be derived from others and can originate other chemical forms of uridine. For instance, $\mathrm{mcm} 5 \mathrm{~S} 2 \mathrm{U}$ results from the addition of $\mathrm{S} 2 \mathrm{U}$ to
mcm5U, and ncm5U leads to the formation of at least the three modified uridines $\mathrm{mcm} 5 \mathrm{Um}$, nchm5U or ncm5S2U (Boccaletto P. et al., 2018);

- We showed that elpc-3 and elpc-1 mutants display different behavioral phenotypes, producing tRNA ${ }^{\text {Ala }}$ (UGC) with different activities;

To see if any of the three $U$ modifications could be involved in chemo-attraction or in the positive or negative imprinting, we used synthetic S2U, mcm5S2U and ncm5U. We first analyzed the behavior of the tut-1 (tm1297) mutants, which lack the tRNA sulfurtransferase activity of the TUT-1 enzyme, thus do not synthesize S2U nor mcm5S2U. Chemo-attraction is impaired by the tut-1 (tm1297) mutation, as shown for CI 300 chemotaxis (Figure 12A). We observed a concentrationdependent restoration of CI 300 response after adding increasing amounts of S2U dilutions (10 $\mu \mathrm{l}$ of 2 to $400 \mu \mathrm{M}$ dilutions) 


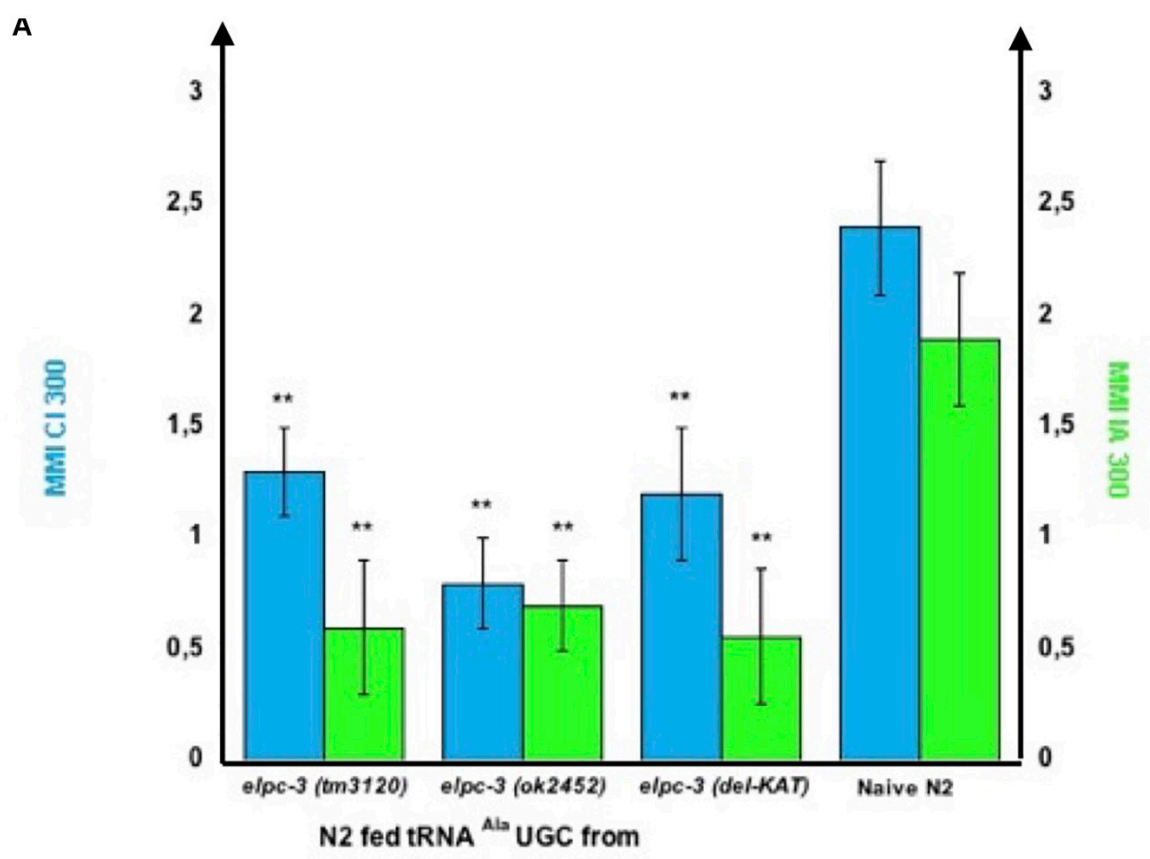

B

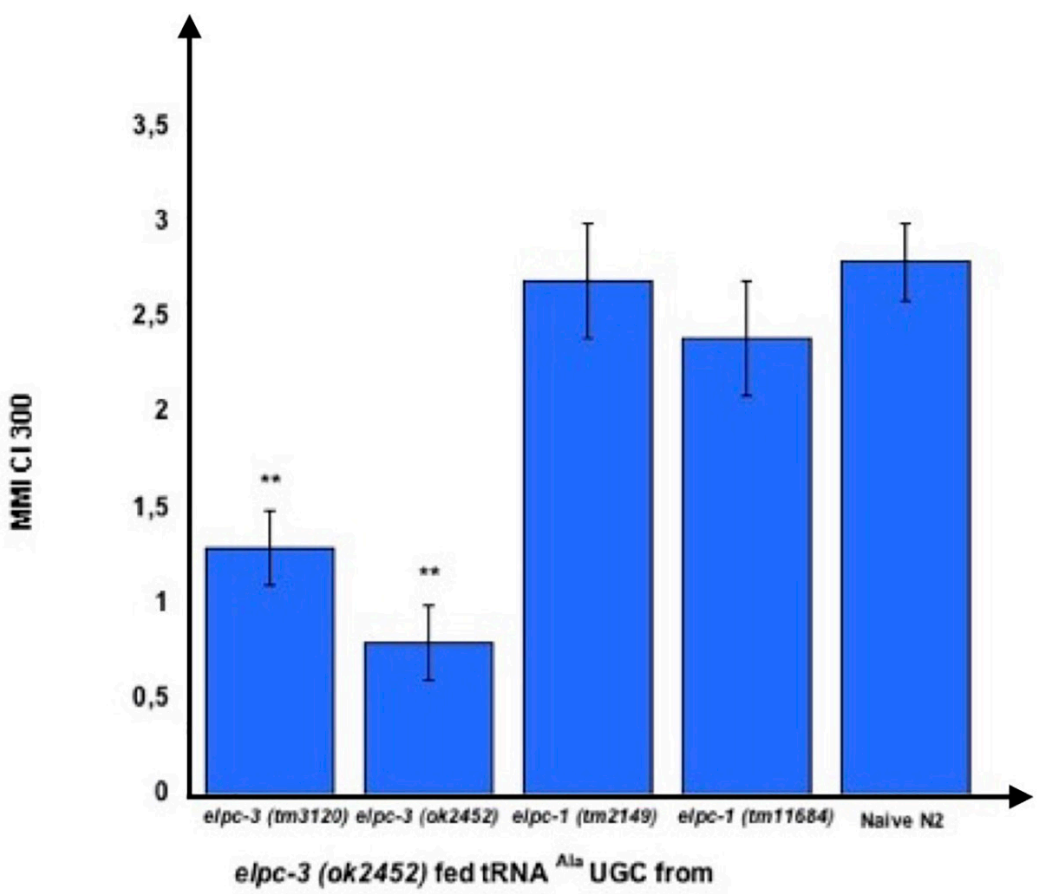

FIGURE 9 | tRNAAlaUGC produced by elpc mutants transfers mutant phenotypes to wild-type via feeding. (A) tRNA ${ }^{\text {Ala }}$ (AGC from the elpc-3 (tm3120), from the elpc-3 (ok2452) and from the KAT domain deleted elpc-3 (del-KAT) mutant worms were microbead-purified as described. Wild-type N2 worms were grown during their whole development in the presence of the indicated elpc-3 mutants tRNAAla UGC or without tRNA for Naive N2. Mean Migration Indices (MMI) in gradients of Citronellol ( $\mathrm{Cl} 300)$ or Isoamyl alcohol (IA 300) were determined at the adult stage as described (experimental repeats $\geq 4$, ${ }^{\star \star} p$-value $\left.<0.01\right)$. (B) Feeding tRNA Ala $\cup G C$ from elpc-1 mutants fully rescues the chemo-attractive defects of elpc-3 mutants. tRNA ${ }^{A l a} \cup G C$ from elpc-3 (tm3120), elpc-3 (ok2452), elpc-1 (tm2149), and from elpc-1 (tm11684) mutant worms were microbead-purified as described. elpc-3 (ok2452) worms were grown during their whole development in the presence of the indicated elpc-3 or elpc-1 mutants tRNA Ala UGC. Mean Migration Indices (MMI) of tRNA fed elpc-3 (ok2452) and of Naive N2 wild-type in Citronellol $(\mathrm{Cl} 300)$ gradients were determined at the adult stage as described (experimental repeats $\geq 4,{ }^{\star \star} p$-value $\left.<0.01\right)$. 


\section{A}

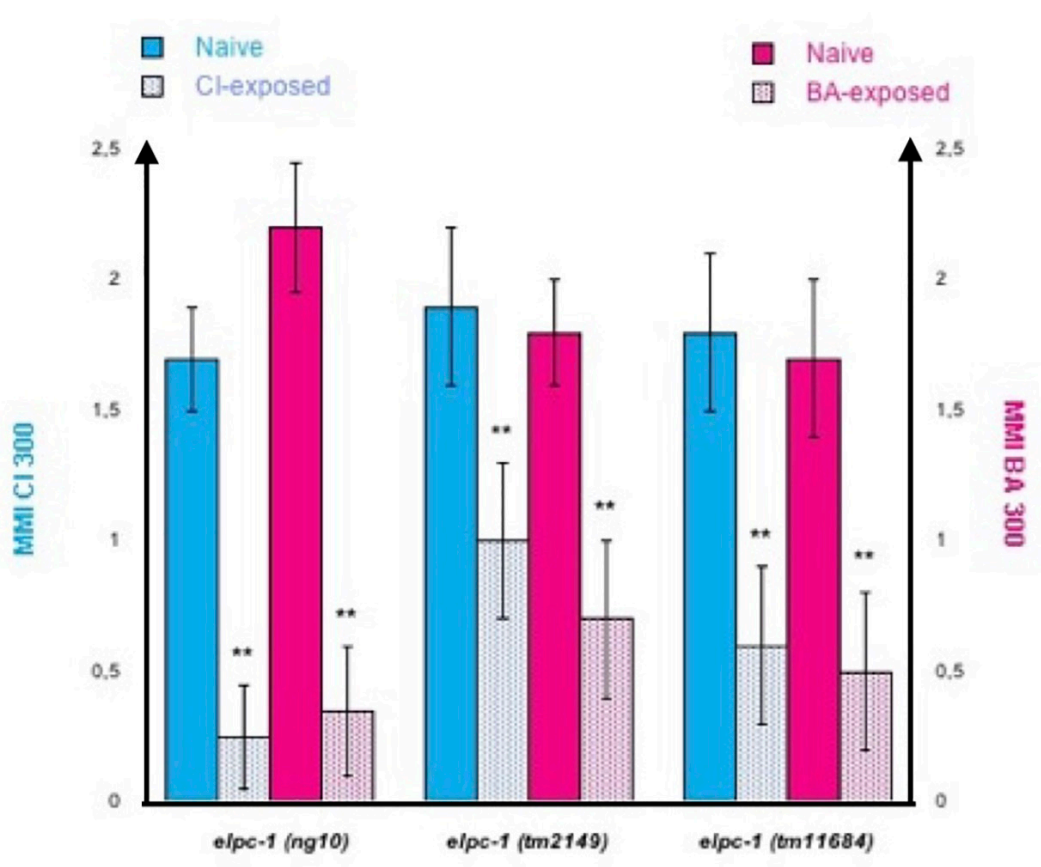

Worm strains

B

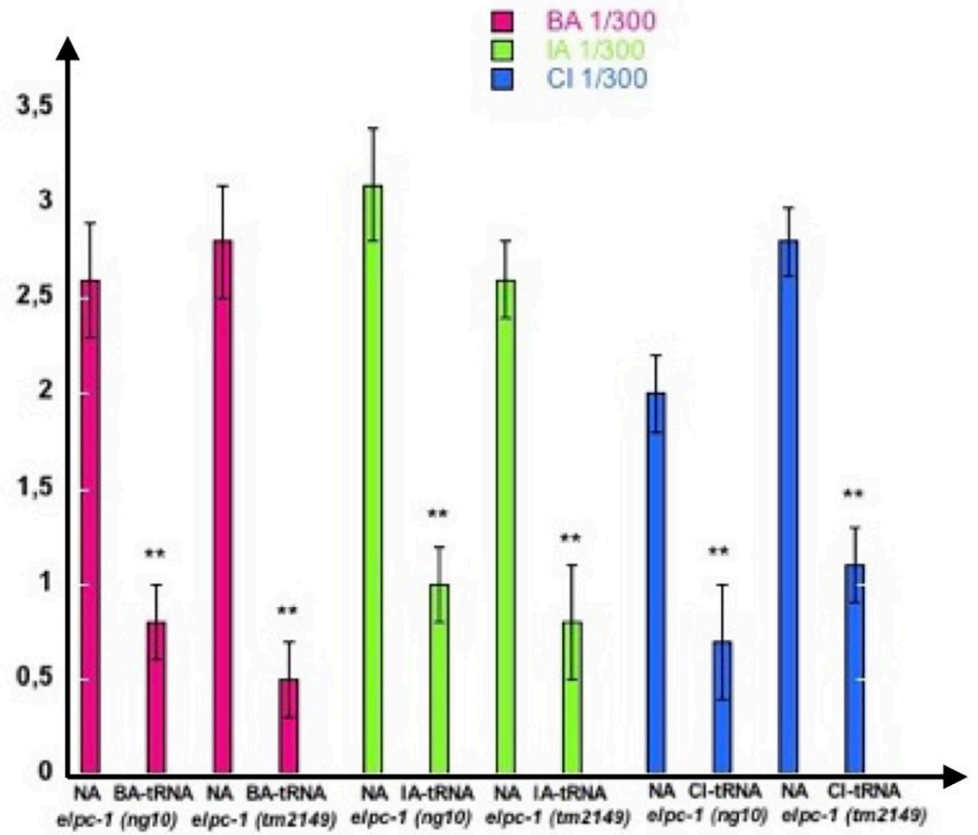

FIGURE 10 | Odor-exposure or feeding odor-tRNAAlaUGC strongly reduce odor-specific chemo-attraction in elpc-1 mutants. (A) elpc-1 (ng10), elpc-1 (tm2149) or elpc-1 (tm11684) mutant worms were either exposed to Cl 1/300, to BA 1/300, or unexposed for control Naive. Mean Migration Indices (MMI) of Naive unexposed, of $\mathrm{Cl}$-exposed and of BA-exposed elpc-1 worms in, respectively, $\mathrm{Cl} 1 / 300$ and BA1/300 gradients were determined at the adult stage as described (experimental repeats $\geq 4$, ${ }^{* *} p$-value $<0.01$ ). (B) elpc-1 (ng10) or elpc-1 (tm2149) worms were fed (or unfed for control naive NA) on BA-tRNA Ala UGC (BA-tRNA), IA-tRNA ${ }^{A l a}$ UGC (IA-tRNA) or Cl-tRNAAla UGC (Cl-tRNA) obtained respectively from BA, IA or Cl-exposed wild-type worms. BA, IA or Cl-tRNA Ala UGC were obtained after microbead purification, gel-separation and gel-elution as described in Figure 4. Mean Migration Indices of Naive unfed (NA) worms were compared to Mean Migration Indices of odor-tRNA fed worms in the respective odor gradients (experimental repeats $\geq 4$, ${ }^{* *} p$-value $<0.01$ ). 

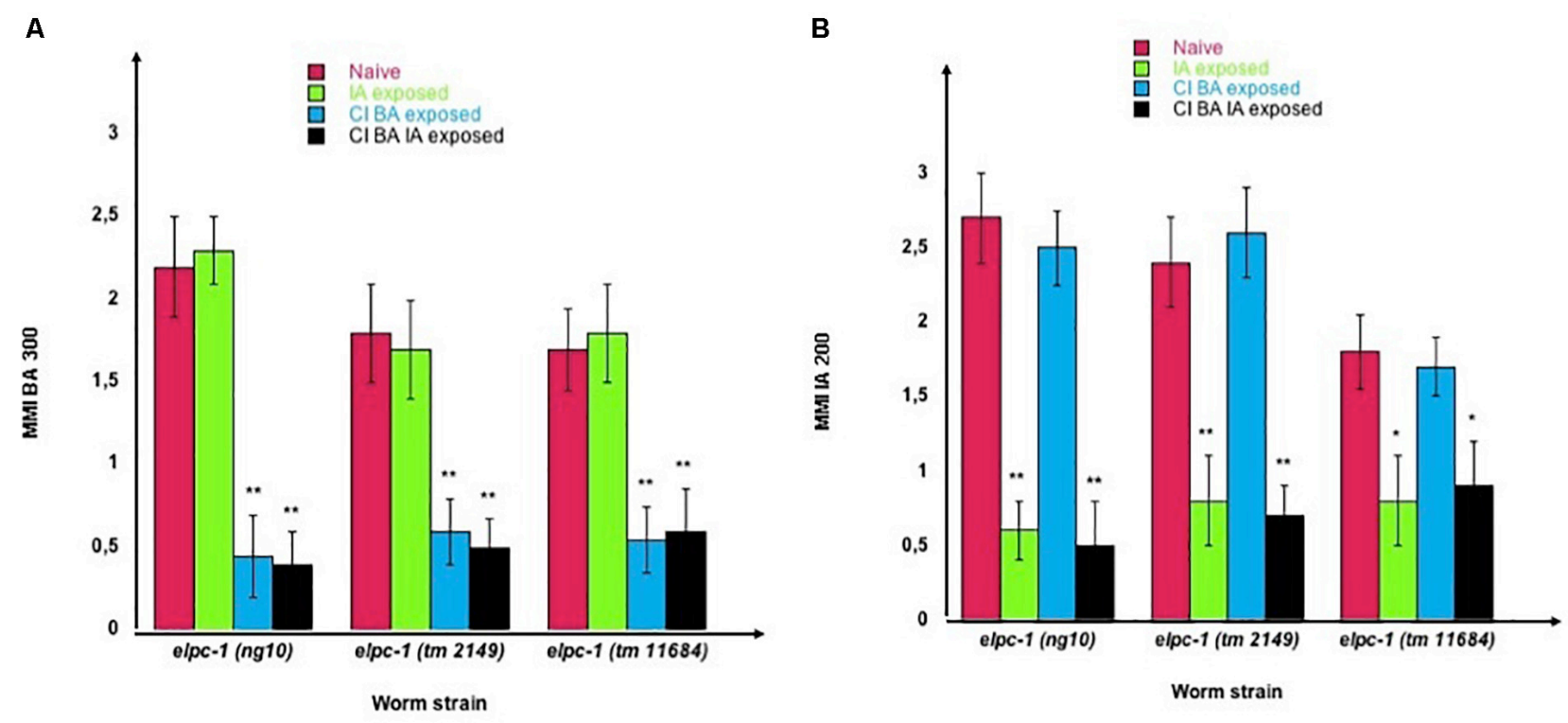

FIGURE 11 | Stable reduction of three odor-specific responses after sequential odor-exposure of elpc-1 mutants. (A) Mean migration Indices (MMI) of Naive unexposed, of IA-exposed, CI BA-exposed and CI BA IA-exposed elpc-1 worms in BA 1/300 gradients were determined at the adult stage, as described (experimental repeats $\geq 4,{ }^{\star *} p$-value $<0.01$ ). (B) Mean migration Indices (MMI) of Naive unexposed, of IA-exposed, Cl BA-exposed and CI BA IA-exposed elpc-1 worms in IA 200 gradients were determined at the adult stage, as described (experimental repeats $\geq 4,{ }^{\star \star} p$-value $<0.01$ ).

to worm food. By contrast, the chemo-attractive defect of the elpc-3 (tm3120) mutants was not restored after feeding the same amounts of S2U, as shown in Figure 12A. Adding S2U does not alter chemo-attraction in wild-type worms (results not shown).

We next assessed imprinting in the tut-1 (tm1297) mutants, and found that, despite a lack of S2U and mcm5S2U, and low chemotaxis responses, these worms positively imprint CI 300, CI200, BA300 and BA 200 (Figure 12B).

These results indicate that a behavioral phenotype linked to the absence of a single identified modified nucleotide (here S2U), can be fully rescued via the addition of the missing nucleotide in worm's environment.

Wild-type tRNAs are believed to have both mcm5S2U and ncm5U, while elpc mutants do not. Since imprinting occurs without S2U nor mcm5S2U, as suggested by the behavior of the tut-1 mutants, then imprinting could, at least partially, involve ncm5U. To test this hypothesis, we fed wt, elpc-3 and elpc-1 mutants on increasing amounts (10 $\mu \mathrm{l}$ of 2 to $400 \mu \mathrm{M}$ dilutions) of mcm5S2U, ncm5U or both together. Adding a single or the two modified Uridines to worm food had no effect on wt or elpc mutants chemo-attraction (not shown). To test if they could influence positive or negative imprinting, we odor-exposed wt and $e l p c-1$ worms in the presence of increasing amounts $(10 \mu \mathrm{l}$ of 1 to $400 \mu \mathrm{M}$ dilutions) of mcm5S2U, ncm5U, or both.

Wild-type imprinting was unchanged in the presence of any amount of one or both modified uridines (not shown). By contrast, we observed clear odor-specific effects of ncm5U in inhibiting the odor-triggered negative imprinting in elpc1 mutants (Figure 13). As already shown (Figure 10), odorexposed elpc-1 mutants acquire a stably inherited odor-specific inhibition of odor responses. The range of $\mathrm{ncm} 5 \mathrm{U}$ required to inhibit elpc-1 negative imprinting varies according to the odors. Adding $10 \mu \mathrm{l}$ of as low as $1 \mu \mathrm{M}$ ncm5U impairs IA 300 imprinting, between 3 and $10 \mu \mathrm{M}$ impairs BA 300 imprinting, while between 30 and $100 \mu \mathrm{M}$ are needed to impair CI 300 imprinting. Upper or lower ncm5U concentrations, outside the respective odor-specific ranges, do not hinder negative imprinting. Figure $\mathbf{1 3}$ data suggest that at least variations of the ncm 5 uridine modification in tRNA ${ }^{\text {Ala }}$ (UGC) could participate in forming the odor-specific codes.

In C. elegans, only two pairs of chemosensory neurons, AWA and $\mathrm{AWC}$, are required for chemotaxis to volatile attractants (Bargmann et al., 1993). The C. elegans genome encodes around 1300 functional seven transmembrane receptors, presumably coupled to G-proteins, called Serpentine Receptors (SR). When expressed in chemosensory neurons, these receptors are thought to interact with odorant molecules and support odor specificity of the chemoattractive responses (Hart and Chao, 2010). The AWC olfactory neurons are responsive to a high number of chemically different molecules that include the three chemoattractants BA, CI and IA used in this study (Bargmann et al., 1993). Only the developing first larval stage L1 can be imprinted by attractive (this study), aversive (Jin et al., 2016), or toxic stress (Gecse et al., 2019) signals present in worm's environment. Environmentresponsive mechanisms must be present at this stage to adapt future worm's behaviors through up or down-regulation of genes expressed in chemosensory neurons. A single chemo-attractive odor signal specifically increases, in wt, or decreases, in elpc1 mutants, adult attraction to a single odorant molecule, while all other AWC-mediated responses remain unchanged. Odorspecificity supports the existence of olfactory signaling insulation within olfactory neurons, as already hypothesized for other forms 

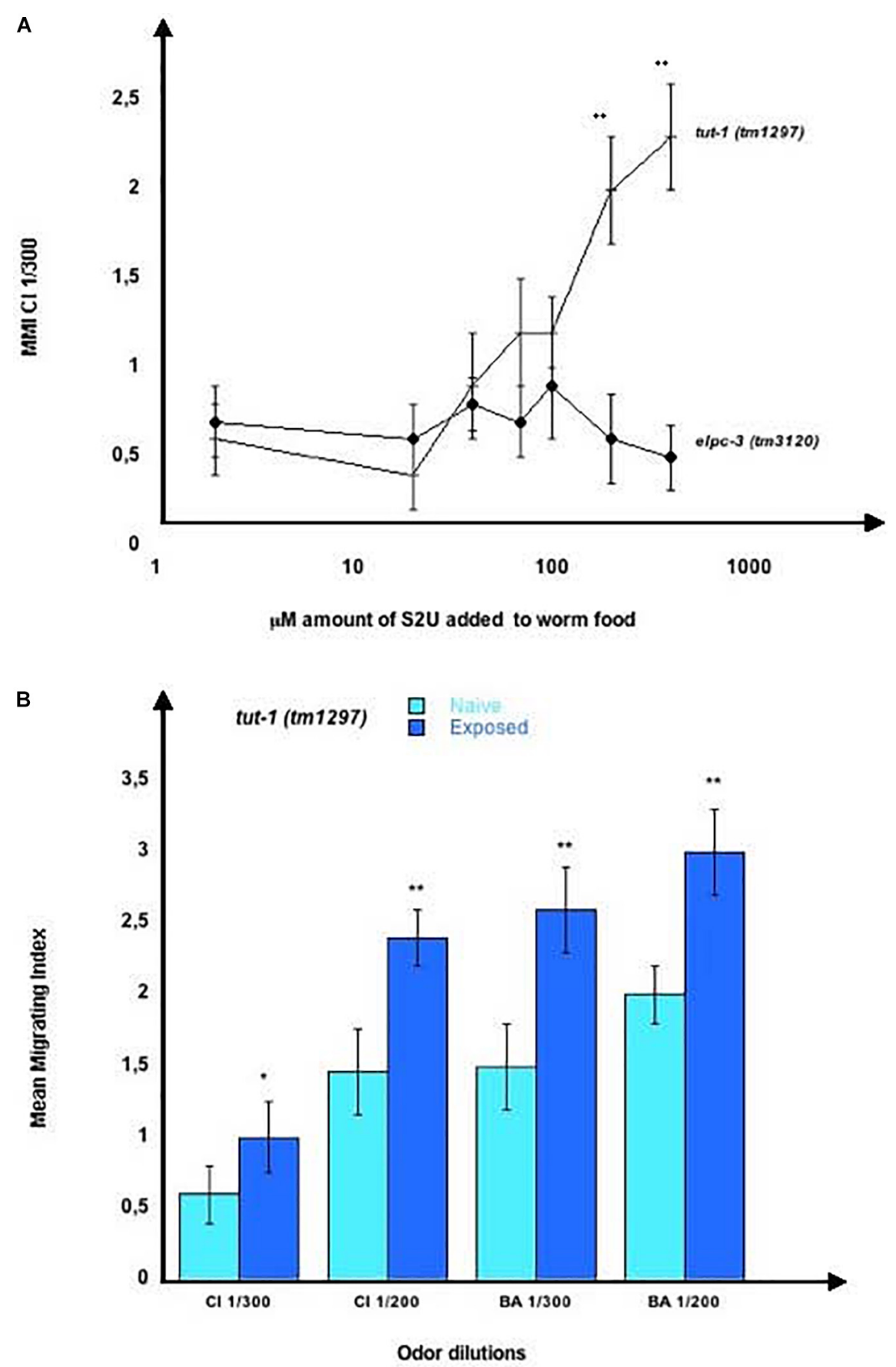

FIGURE 12 | Chemo-attractive behavior of the tut-1 (tm1297) mutant worms. (A) Mean migration indices of tut-1 (tm1297) and elpc-3 (tm3120) mutant worms in a $\mathrm{Cl} 1 / 300$ gradient in the presence of increasing concentrations of 2-thiouridine (S2U). The $20 \mathrm{mM}$ stock of $\mathrm{S} 2 \mathrm{U}$ in $\mathrm{H} 2 \mathrm{O}$ was diluted to obtain the indicated concentrations, of 2 to $400 \mu \mathrm{M} .10 \mu \mathrm{l}$ of each dilution were added to the bacterial spot (worm food) before the addition of worm eggs. MMl were determined at the adult stage, as described (experimental repeats $\geq 4,{ }^{\star \star} p$-value $<0.01$ ). (B) tut-1 (tm1297) worms were unexposed for Naive or Exposed to, respectively, Cl 1/300, Cl 1/200, BA 1/300 or to BA 1/200. Mean Migration Indices in the respective odor-dilutions gradients were determined at the adult stage, as described (experimental repeats $\geq 4,{ }^{\star} p$-value $<0.05,{ }^{* *} p$-value $<0.01$ ).

of odor-induced adaptation in C. elegans (O'Halloran et al., 2009; Juang et al., 2013).

Naive unexposed elpc-1 worms are odor-responsive, suggesting they express functional chemo-receptors. Although the outcome of single odor-exposure is to decrease chemoattraction irreversibly, naive elpc-1 mutants might still produce odor-specific tRNA ${ }^{\text {Ala }}$ (UGC) upon odor-stimulation. We hypothesized that the lowered odor-responses displayed by 


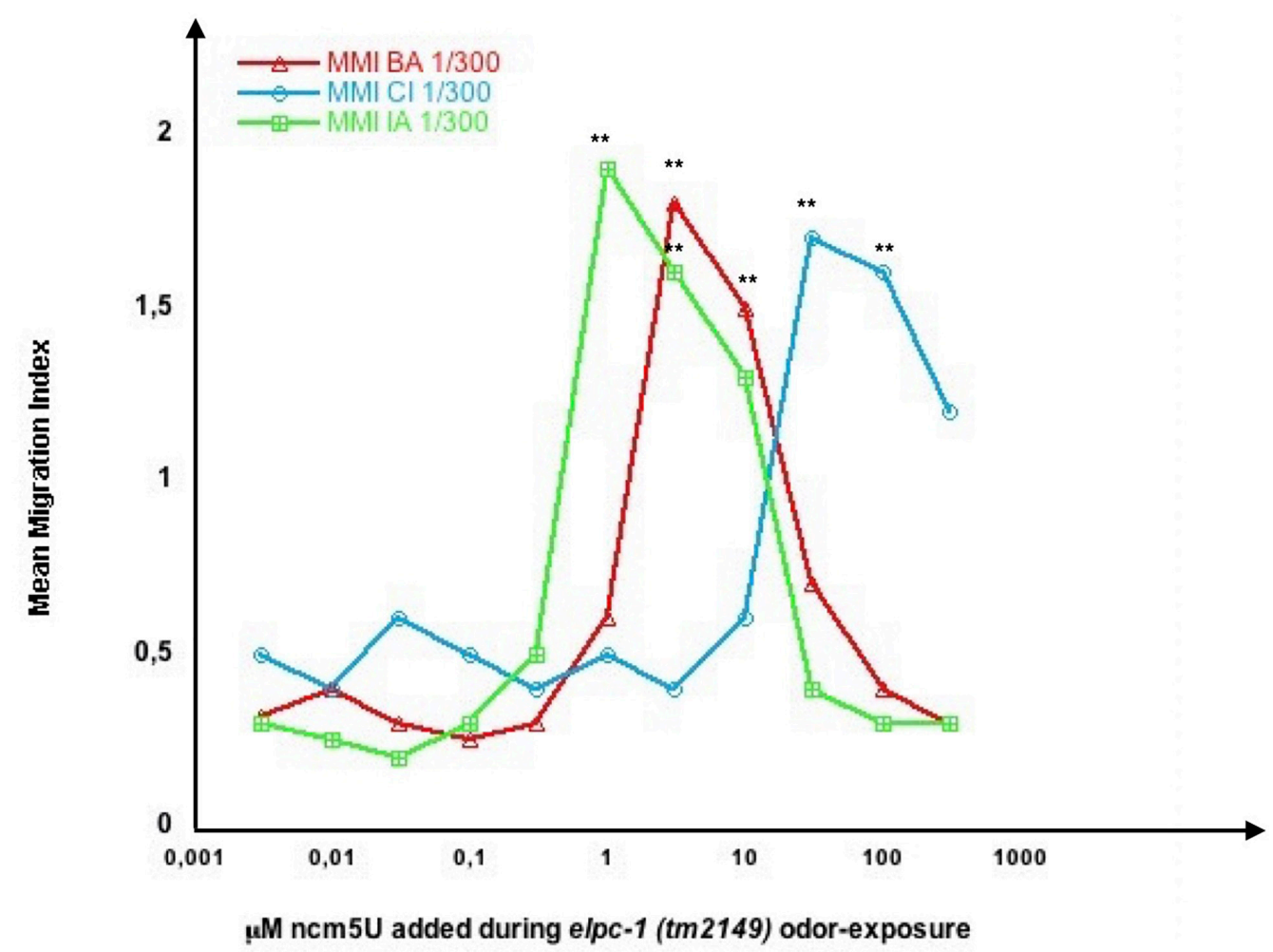

FIGURE 13 | Odor-specific ranges of ncm5 modified Uridine impair odor-specific negative imprinting in elpc-1 mutants. elpc-1 (tm2149) mutant worms were odor-exposed to either BA 1/300, Cl 1/300 or IA 1/300 in the presence of increasing amounts of 5-carbamoylmethyluridine (ncm5U). The indicated concentrations of ncm5U, from 0.004 to $400 \mu \mathrm{M}$, were obtained by dilution of the $20 \mathrm{mM}$ stock solutions in Tris $0.3 \mathrm{M} \mathrm{pH} 8.10 \mu \mathrm{l}$ of each dilution were added to the bacterial spot (worm food) before the addition of worm eggs. Mean migration Indices (MMI) in the respective BA, Cl or IA odor gradients were determined at the adult stage, as described (experimental repeats $\geq 4,{ }^{* *} p$-value $<0.01$ ).

negatively imprinted elpc-1 worms (Figures 10, 11) could be due to lowered or null expression of the respective olfactory receptors. Without receptors, odors would not lead to the production of odor-modified tRNA Ala (UGC). Naive elpc-1 (ng10) mutants are called $\mathrm{CI}+$ or $\mathrm{BA}+$, while elpc-1 (ng10) that carry negative imprints of $\mathrm{CI}, \mathrm{BA}$ or for $\mathrm{CI}$ and $\mathrm{BA}$ are respectively called CI-, BA-, or CI-BA-. We fed N2 wt on tRNA ${ }^{\text {Ala }}$ (UGC) purified from naive, $\mathrm{CI}+$, $\mathrm{CI}-, \mathrm{BA}+$, BA-, and CI-/BA- elpc-1 (ng10) worms, as indicated in Figure 14.

As shown in Figure 14, tRNA $^{\text {Ala }}$ (UGC) from odor-exposed enhances chemo-attraction of wt worms, compared to tRNA ${ }^{\text {Ala }}$ (UGC) from naive unexposed. By contrast, tRNA ${ }^{\text {Ala }}$ (UGC) from worms bearing a CI- negative imprint or a BA- negative imprint, do not, respectively, enhance CI or BA chemoattractive responses of wt worms. As expected, elpc-1 (ng10) with a double CI-/BA- negative imprint do not enhance wt attraction to $\mathrm{CI}$ nor $\mathrm{BA}$.

Our data suggest elpc-1 worms produce a wt form of tRNA ${ }^{\text {Ala }}$ (UGC), able to rescue the elpc-3 chemotaxis phenotype.

From the results shown in Figure 14, we can conclude that naive never-exposed elpc-1 mutants produce the odor-specific tRNA $^{\text {Ala }}$ (UGC) after odor-exposure, able to transfer positive imprinting to wild-type. However, odor-stimuli no longer trigger the production of odor-specific tRNA ${ }^{\text {Ala }}$ (UGC) in negatively imprinted elpc-1 worms. Whether or not negative imprinting is due to a down-regulation of chemo-receptor expression remains to be demonstrated.

Attractive odor stimuli activate an AWC-specific olfactory transduction pathway made of several $G$ proteins, the DAF11/ODR-1 guanylyl cyclase, and the TAX-2/TAX-4 cGMP-gated channel. Worms with mutations inactivating members of this pathway are defective for all AWC-mediated chemosensory responses, including the responses to the three attractive odorants used in this study (Bargmann, 2006).

We asked whether a functional AWC olfactory transduction pathway is required for the synthesis of odor-tRNAs. We purified tRNA ${ }^{\text {Ala }}$ (UGC) from CI-exposed worms harboring the odr-1 (n1936), the tax-2 (p671) or the tax-4 (p678) mutations, respectively, inactivating the ODR-1 guanylyl cyclase, the TAX-2 or the TAX-4 subunits of the cGMP-gated channel. Wild-type and elpc-1 (ng10) mutants were then fed on these tRNAs, to compare their imprinting activities (Figure 15).

As already shown, feeding on tRNA ${ }^{\text {Ala }}$ (UGC) from CIexposed wt increase CI MMI in wt (positive imprinting), and decrease CI MMI in elpc-1 (ng10) (negative imprinting). By contrast, none of the three CI-exposed olfactory transduction mutants produce a tRNA ${ }^{\mathrm{Ala}}$ (UGC) able to transfer a positive 


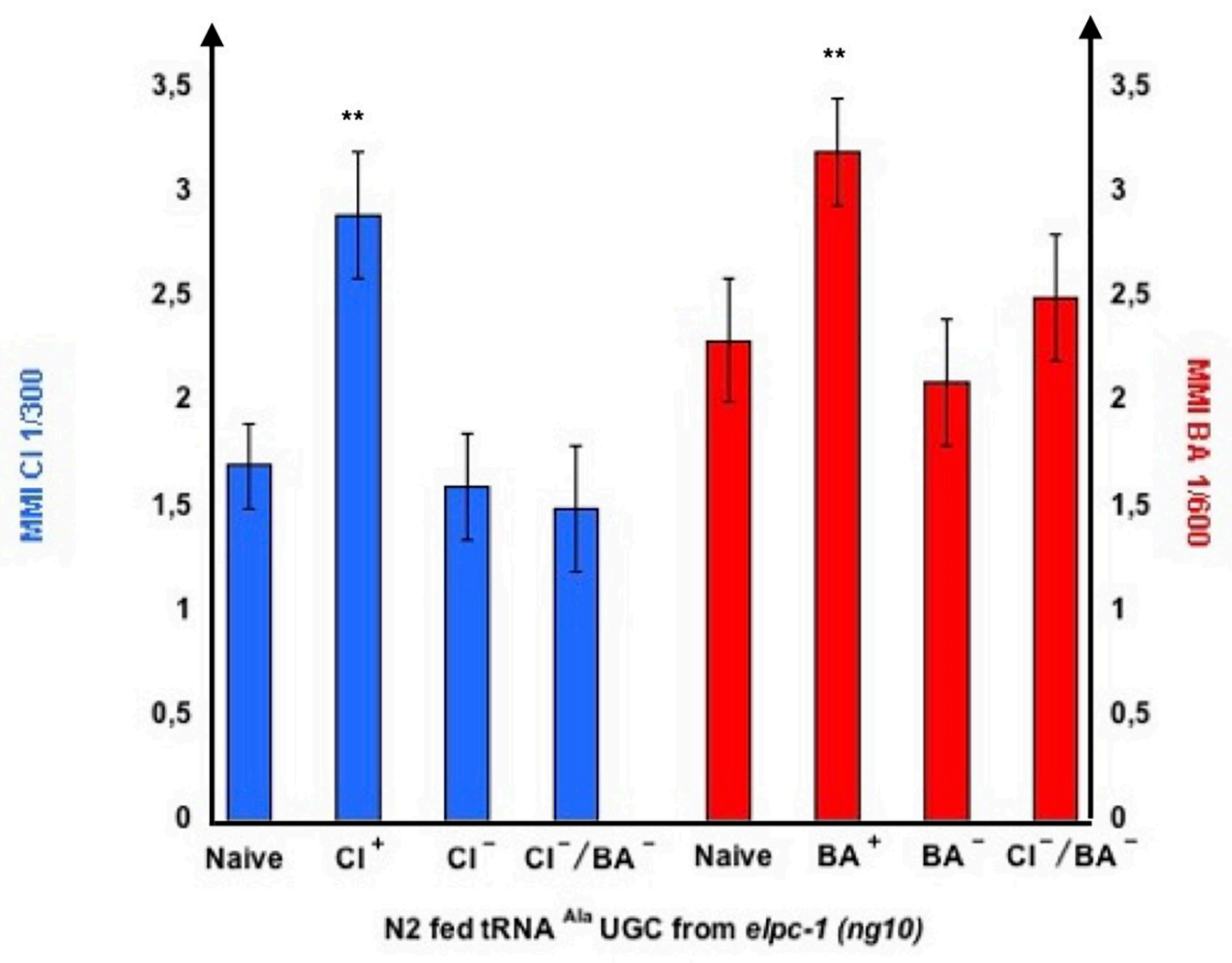

FIGURE 14 | tRNAAla UGC from negatively imprinted elpc-1 (ng10) mutants do not transfer imprinting to naive. Stable reduction of Cl response, BA response or Cl and BA responses were obtained in elpc-1 (ng10) after odor-exposure or after odor-tRNA feeding, as described in Figure 11. Naive unexposed elpc-1 (ng10) worms are annoted $\mathrm{Cl}+$ and $\mathrm{BA}+$. elpc-1 (ng10) worm populations with stable reduction of attraction to $\mathrm{Cl}$, to $\mathrm{BA}$ or to $\mathrm{Cl}$ and $\mathrm{BA}$ are respectively annoted $\mathrm{Cl}-$, $\mathrm{BA}$-, or Cl-/BA-. N2 wt were fed on tRNA ${ }^{\text {Ala }}$ UGC respectively purified from elpc-1 (ng10) worms (left to right): Naive, $\mathrm{Cl}$-exposed, stable $\mathrm{Cl}$ - $\mathrm{Cl}$-exposed, stable $\mathrm{Cl}$-/BACl-exposed, Naive, BA-exposed, stable BA- BA-exposed, and stable Cl-/BA- BA-exposed. MMI to Cl 1/300 and to BA 1/300 were determined as described (experimental repeats $\geq 4,{ }^{\star \star} p$-value $<0.01$ ).

CI-imprint to naive wt or a negative imprint to elpc-1 (ng10) (CI-exposed odr-1, tax-2, and tax-4).

Taken together, the results indicate that imprinting requires chemo-receptors to be expressed and coupled to a functional odor-transduction pathway, as early as the imprinting L1 critical stage (Figures 14, 15). They also support the idea that the odor-specific production of tRNA ${ }^{\text {Ala }}$ (UGC) occurs downstream of odor-signaling.

\section{DISCUSSION}

Our data indicate that both the innate and the experiencemodulated chemo-attractive behaviors would be under the control of a single tRNA molecule. To our knowledge, this is the first report demonstrating a behavior is under the control of a tRNA.

The $\mathrm{tRNA}_{\text {Ala }}$ (UGC) would carry specific chemical codes required to acquire chemo-attractive responses, and transmit odor-specific information. As other RNAs, tRNAs can adopt many chemical forms by combining more than 160 chemically modified bases (Boccaletto P. et al., 2018). The combinatorial calculation indicates that even if just a fraction of the nucleotides is chemically modified, the number of possible combinations remains very high. Theoretically, choosing 10 base modifications (m) out of 100 (n) already creates more than $10^{13}$ possible combinations, according to the $(n+m-1) ! /((n-1) ! * m$ ! equation. To understand how such a huge potential of chemical diversity is translated into functional diversity represents a considerable challenge.

Although mature tRNAs mainly control protein translation, tRNAs can be fragmented into smaller RNA molecules. tRNA fragments, tRFs, are an emerging class of non-coding RNAs that may, besides translation, control several other important biological functions (Megel et al., 2015; Pliatsika et al., 2018).

The $3 \mathrm{D}$ structure and base modifications contribute to the high stability of mature tRNAs (Engelke and Hopper, 2006). It would be interesting to analyze if and how mature tRNAs could be processed after contact with the bacterial lawn (worm food) and after ingestion and diffusion from intestinal cells to other tissues, including germ-line cells. It has been already shown that endogenous RNAi in the chemosensory neuron AWC promotes odor-specific adaptation in adult C. elegans (Juang et al., 2013). If Dicer-independent mechanisms can generate tRFs, the tRNA 


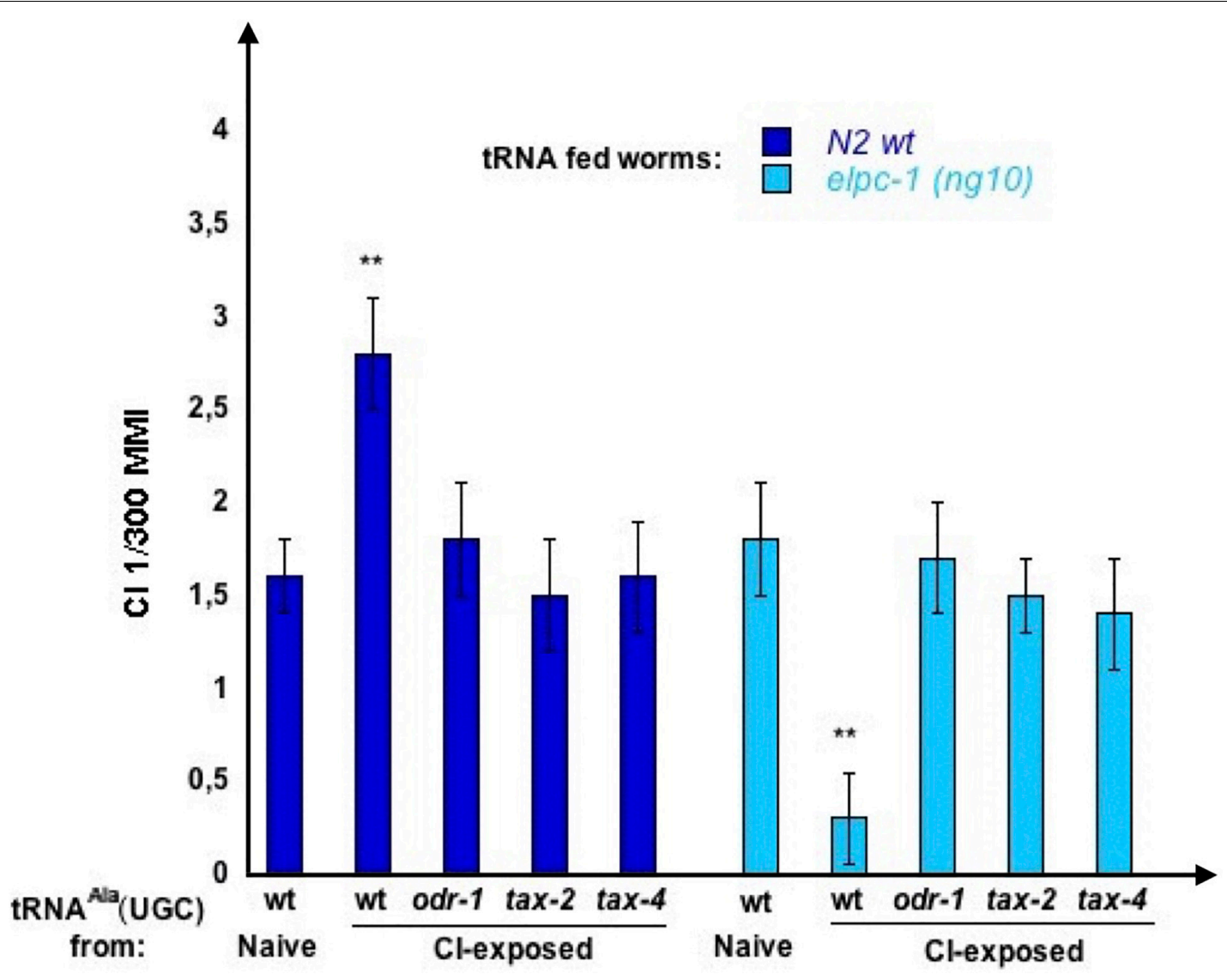

FIGURE 15 | Olfactory transduction mutants do not produce odor-imprinting tRNA Ala (UGC) after odor-exposure. tRNA ${ }^{\text {Ala }}$ (UGC) was microbeads purified from Naive unexposed, Cl 1/300-exposed wt N2 worms, Cl 1/300-exposed odr-1 (n1936), Cl 1/300-exposed tax-2 (p671), and from Cl 1/300-exposed tax-4 (p678) mutant worms. Naive wt and naive elpc-1 (ng10) worms were fed on these tRNA Ala (UGC). Mean Migration Index to $\mathrm{Cl} 1 / 300$ was determined at the adult stage as described $\left({ }^{* *} p\right.$-value $\left.<0.01\right)$.

double-stranded stretches could be processed via the well described Dicer-dependent small non-coding RNA pathways. If it is the case, genetic inactivation of these pathways would impair the $\mathrm{tRNA}_{\mathrm{Ala}}$ (UGC) triggered biological activities described in this paper. Moreover, comparative RNA sequencing after tRNA $_{\text {Ala }}$ (UGC) feeding may help to identify which small RNAs eventually originated from tRNA processing could trigger the epigenetic changes.

Each nucleotide chemical modification requires the activity of specific enzymes, called writers. So far, no C. elegans tRNA writers are known, except TUT-1 and the Elongator complex, both modifying Uridines, respectively, into S2U, mcm5S2U and ncm5U. Through the addition of different amounts of these three modified Uridines to worm food during odor-exposure, we identified ncm5U as being part of the odor-specific codes carried by $\mathrm{tRNA}_{\mathrm{Ala}}$ (UGC) (Figure 13). Providing worms with different amounts of ncm5U however only impairs, thus odor-specifically, the negative olfactory imprinting in elpc-1 mutants but does not affect the positive imprinting in wild-type.

We hypothesized that specific patterns of base modifications would stand for specific biological activities. Transfer and inheritance may rely on yet unknown reading mechanisms, able to recognize the modification patterns, and on copying mechanisms, able to precisely reproduce those.

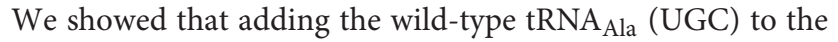
food of elpc-3 mutants rescues their chemo-attractive defects (Figure 8). The rescue could rely on the compensation of the lacking modified $U$ by those carried by the wt tRNA.

However, adding the "defective " elpc-3 tRNA Ala $_{\text {(UGC) to }}$ wt worms phenocopy the elpc-3 behavioral phenotype (Figure 9). In this case, compensation for lacking modifications cannot account for the transferred phenotype, suggesting phenocopy could be rather based on recognition and reproduction of the " defective » modification pattern in the mutants. The mechanisms by which epitranscriptomic codes are written, read, copied or erased, remain elusive. Analyzing the activity of a tRNA Ala $_{\text {(UGC) }}$ made by wild-type worms fed on tRNA $_{\mathrm{Ala}}$ (UGC) made by known modification mutants, as $t u c-1$, and inversely, could provide some further insights.

We report that deletions in the elpc-3 gene do not have the same behavioral effects as the elpc-1 gene deletions. Our observations suggest that the catalytic sub-unit ELPC-3 modifies tRNAs in the absence of a functional ELPC-1, while ELPC-1 is required for imprinting. They challenge previous reports in 
which the inactivation of any elongator sub-unit produced the same phenotypes. However, the structural organization and the respective biochemical functions of the C. elegans Elongator subunits are unknown. Whether the multiple elongator functions only rely on its tRNA modifying activity, or other biochemical activities of its sub-units, is still debated. As indicated in WormBase $^{1}$, the comprehensive resource for nematode research, $e l p c-1$ genetically interacts with chaf-2 and dhc-1. CHAF2 is an ortholog of the human chromatin assembly factor CHAF1B, involved in nucleosome assembly, linking newly synthesized histones deposition to DNA replication (Sauer et al., 2018). DHC-1, the unique C. elegans cytoplasmic dynein 1 $\mathrm{HC}$, controls microtubule dynamics and is involved in many essential biological processes, including neuron development and meiotic spindle orientation (O'Rourke et al., 2007). These two interactions could be particularly relevant to the elpc-1 mutant phenotype described here, which associates tRNA metabolism, epigenetic reprogramming and neuro-development.

Nematodes respond to many volatile odorants but have only three pairs of olfactory neurons, AWA, AWB and AWC, suggesting each neuron would express a large number of odor-sensitive chemo-receptors. It has been shown that the type of behavioral response elicited by an odorant is not specified by the chemo-sensory receptors, but by the olfactory neuron in which they are expressed and activated (Troemel et al., 1997). Our results suggest chemo-receptors for attractive cues are expressed and already functional, i.e., coupled to the olfactory transduction pathway, at the first stage of larval development. The way each worm's chemoresponsive neuron expresses specific sets of receptors and represses the expression of all others is still unknown. In mammalian species, each olfactory neuron expresses only one allele of a member of the olfactory receptor genes family; the precise mechanisms by which such monogenic monoallelic receptor choice are achieved are however not fully understood. These mechanisms include nuclear compartimentalization, repression/derepression through epigenetic marks as those made by the chromatin-modifying enzyme Lysine demethylase 1 (LSD-1) and stabilization of functionally expressed receptors by the odor-signaling pathway (reviewed in Degl'Innocenti and D’Errico, 2017).

tRNAs and tRNAs genes control translational (Avihu et al., 2013; Kirchner and Ignatova, 2015) and transcriptional finetuning (Pratt-Hyatt et al., 2013; Woolnough et al., 2015; 13Kirkland and Kamakaka, 2015). Importantly for epigenetic inheritance, tRNAs also control the plasticity of genome architecture via the dynamic positioning of nucleosomes and the modulation of chromatin domain boundaries (McFarlane and Whitehall, 2009; Talbert and Henikoff, 2009; Raab et al., 2012).

In this work, we produced worm strains with stably inherited non-genetic alterations of odor-specific responses, compared to wild-type. Increased or decreased odor-specific attraction may rely on the differential expression of odor-specific receptors. Through combinatorial epigenetic modifications, the nuclear localization of their genes may evolve, moving from either active

${ }^{1}$ wormbase.org or repressed chromatin domains (Armelin-Correa et al., 2014; Evans et al., 2016).

The chemo-receptors expression pattern in C. elegans sensory neurons is unstable as it can be altered by life history and external conditions (Vidal et al., 2018). Worms are innately attracted by many volatil molecules, however only within a specific range of dilutions. The mechanisms that specifically direct attraction to each odor-specific dilution might result from the sensory neuron distribution and the differential expression levels of specific sets of chemo-receptors.

Identifying the putative chemo-receptor genes involved in an odor-specific response is a prerequisite to delineate further the epigenetic mechanisms that would regulate expression patterns and levels. The stably odor-specifically imprinted worms described here, displaying permanent higher or lower responses to single or to multiple odorants, compared to wild-type naive, might significantly facilitate the identification of these genes.

This work highlights that a specific tRNA and the multifunctional Elongator complex have a key role in translating environmental inputs into behavioral changes.

It sets the stage to elucidate how environmental information, here olfactory cues, could regulate secondary and tertiary tRNA structures, which would, in turn, influence worm behavior.

Due to the growing number of human diseases linked to RNA modification defects (Sarin and Leidel, 2014), understanding the biological significance of the epitranscriptome is a major issue. Recent findings strongly indicate that RNA modifications play dynamic regulatory roles analogous to the epigenetic changes of DNA and histone proteins. Understanding the function and mechanisms of the dynamic RNA modifications, or epitranscriptomic, represents a new challenge at the frontier between different disciplines. Reversible RNA modifications add a new dimension to the developing picture of post-transcriptional regulation of gene expression. This new dimension awaits integration with transcriptional regulation to decipher the multi-layered information that controls a plethora of biological functions. Understanding how non-coding transcriptomes are modified in response to environmental changes and how these modifications impact germ-line cells and translate into inherited phenotypes thus represents an important challenge (Schimmel, 2018).

Olfactory imprinting serves hatchling attachment and adult homing to the native chemo-sensory environment in many animal species (Grassman et al., 1984; Dittman and Quinn, 1996; Horn, 2004; Gerlach et al., 2007, 2019).

As we describe here for the nematode, multigenerational imprinting of the same olfactory cues would lead to stable inheritance. Through a process of (epi)genetic assimilation, different animal populations of the same species may have acquired specific patterns of chemo-attractive responses, only depending on their local living environments. This kind of " cultural " differentiation, through the stable assimilation of responses to external challenges, may be instrumental to the evolution of innately expressed behaviors (Jablonka and Lamb, 2015).

Our working hypothesis is modeled in Figure 16. In C. elegans, the first post-hatching hours are highly receptive to 


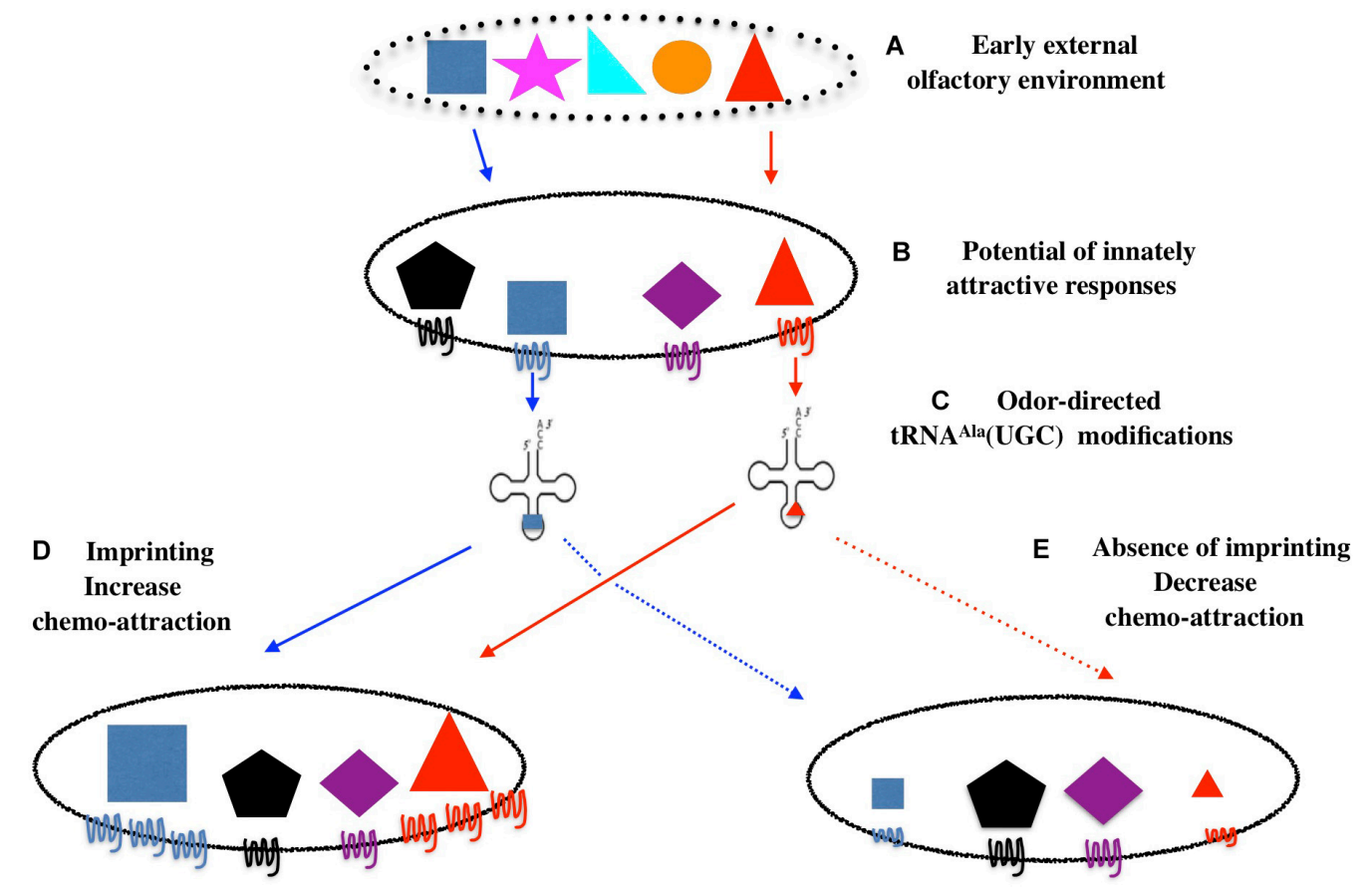

FIGURE 16 | Imprinting is an innate behavior that sanction innately encoded chemo-attraction and early environment adequacy. The olfactory environments in which animals come to life contains a number of chemicals (A). Worms are innately attracted by some of these chemicals, here represented by the blue square and the red triangle (B). (C) Attractive odors trigger the production of tRNA ${ }^{\text {Ala }}$ (UGC) carrying odor-specific information. (D) Imprinting will increase worm attraction to odorants that are both present in the early environment and encoded as innately attractive. (E) In the absence of imprinting, as in elpc-1 mutants, odorants will stably decrease the innate worm responses for these odorants.

environmental conditions (Hall et al., 2010; Jin et al., 2016; Hong et al., 2017; Gecse et al., 2019). Imprinting chemo-attractive cues is an innate behavior taking place during this period of development. Its biological function would be to confirm that olfactory cues present in the early environment are indeed encoded as innately attractive and will be potentially rewarding for the future worm life and for its progeny.

An innate frame would encode and program the potential of C. elegans chemo-attractive responses. Qualitative (to which chemicals) and quantitative (attractive strength) responses are stably inherited over generations in stable environments. As environment changes, it may or not contain cues encoded as innately attractive. If it does not, the spectrum of behavioral responses will not change as it relies on the innate program.

If it does, external olfactory cues will interact with the chemoreceptive elements set up to transduce chemo-attraction. Such interactions would trigger odor-specific labeling of tRNA ${ }^{\text {Ala }} \mathrm{UGC}$, using the combinatorial potential of tRNA bases modifications. In this diffusible form, odor-specific information can move internally, from neuronal to, eventually, germ-line cells, and, as we show here, transfer olfactory imprints from experienced to naive animal. Such diffusible mnemonics would be a way to encode, transfer and communicate experience without modifying the neuronal synaptic network. Moreover, they could support the transfer of experience in biological organisms with a low number or without neurons (Boussard et al., 2019). The reading mechanism able to translate epitranscriptomic codes into unstable or stably inherited epigenetic codes remains to be discovered. By enhancing their attractiveness, the role of imprinting would be to confirm that the newly encountered cues are well assessed as attractive by the innately encoded program.

In the case environmental signals and innate program coincide, attraction can not be confirmed in imprinting defective animals, as we showed here for elpc-1 deleted mutants. Without confirmation by the imprinting process, worms would be definitely desensitized and lose innate attraction to the specific cues they have been exposed to. Imprinting is thus an innate behavior that supports the plasticity of the innate directory of behaviors.

\section{MATERIALS AND METHODS}

\section{Modified Uridines}

S2 Uridine (S2U) was obtained from Jena Bioscience (Germany) and diluted in water. 5-methoxycarbonylmethyl 2-thiouridine (mcm5S2U) and 5-carbamoylmethyluridine (ncm5U) were custom-synthesized by Jena Bioscience and diluted from $20 \mathrm{mM}$ stocks in Tris $0.3 \mathrm{M} \mathrm{pH} 8$.

\section{Strains and Culture Conditions}

We used the wild-type $C$. elegans reference strain N2, unless otherwise stated. 
The sid-2 (qt13) mutant was obtained from Dr. C. Hunter. The 375bp deletion in the elpc-3 gene called here delKAT was produced by Nemametrix, Inc. (Eugene, OR, United States) using the CRISP-sdm transgenic method. The deletion was confirmed by PCR/RE and sequencing. The elongator mutants elpc-1 (ng10) [or ikap-1 (ng10)] were from Dr. J. A. Solinger. The elpc-3 (tm3120), elpc-1 (tm2149), elpc-1 (tm11684) and tut-1 (tm1297) knockouts were generated by the National Bioresource Project, Tokyo, Japan, which is part of the International C. elegans Gene Knockout Consortium. Other strains used in this study were provided by the Caenorhabditis Genetics Center (CGC) funded by the National Institutes of Health (NIH) Office of Research Infrastructure Programs (P40 OD010440). Worms were cultured on E. coli OP50 at $20^{\circ} \mathrm{C}$ using standard protocols.

\section{Odor-Exposure for Olfactory Imprinting}

Benzaldehyde, ${ }^{\circledR}$-citronellol or Isoamyl alcohol (Sigma-Aldrich) were diluted as described in water. Odor-exposures were done by suspending a $4 \mu \mathrm{l}$ drop of these dilutions on the lids of worm culture dishes at least during $24 \mathrm{~h}$ from the egg stage at $20^{\circ} \mathrm{C}$, covering the critical plasticity period corresponding to the first $12 \mathrm{~h}$ of post-hatch development (Remy and Hobert, 2005).

\section{Chemotaxis Assay}

The chemotaxis assay used in this study is schematically outlined in the Supplementary Figure Method 1. It is based on the population chemotaxis assay initially described by $\mathrm{C}$. I. Bargmann (2006). Several modifications were made in the procedure and the chemotaxis index calculation. Changes aimed at more accurately compare the chemo-attraction of worm populations to moderately attractive odorants (as the dilutions of attractive odorants used in this study), using 20 adult worms per condition.

$40 \mathrm{ml}$ of low-salt $\left(1 \mathrm{mM} \mathrm{Ca}^{++}, 1 \mathrm{mM} \mathrm{Mg}^{++}, 5 \mathrm{mM} \mathrm{KP04}\right)$ agar $(20 \mathrm{~g} / \mathrm{l})$ were poured in $12 \times 12 \mathrm{~cm}$ square plates. Assay plates were allowed to dry at room temperature for at least three days before use. Worms were individually transferred from culture dishes to the middle of the square assay plates. To establish a homogeneous odor gradient, so that all worms were submitted to the same olfactory stimulus, 3 drops of odordilution ( $4 \mu \mathrm{l}$ each) were suspended on the lids at one side, each placed at a distance of $3 \mathrm{~cm}$ from the others. On the opposite side of the lids were placed $3 \times 4 \mu$ l of water. 6 drops of $4 \mu \mathrm{l}$ of $1 \mathrm{M}$ $\mathrm{NaN} 3$ were added at both sides of the agar plate to immobilize animals that reached the edges.

At time 0, 20 worms were placed on the middle line of the squared plate, every worm being at a distance of $6 \mathrm{~cm}$ from odor sources. Assays performed on squared plates allowed the indexation of all worm positions between the starting line (time 0 , position $0 \mathrm{~cm}$ ) and the odor source (position $+6 \mathrm{~cm}$ ), usually four times at 10, 20, 30 and $40 \mathrm{~min}$ from time 0 . The mean value of all indexed positions (in $\mathrm{cm}$ from the starting line) of each of the 20 worms represented the Mean Migration Index (MMI). Each experiment shown in the paper was performed at least 4 times. MMI (Means \pm S.E.) values were compared using unpaired data with unequal variance Student $t$-tests performed with the
KaleidaGraph program. Assays were always performed so as to compare synchronized worm populations.

\section{RNAs Fractionation and tRNA Purification}

The large and small RNAs ( $<200 \mathrm{nt}$ ) were separated using the NORGEN RNA Purification Kit (Norgen Biotek Corp). Small RNAs were further size-fractionated on 3.5\% low-melting agarose (Nusieve GTG) gels. RNA was quantified with the Nanodrop 2000 (ThermoScientific). To purify C. elegans transfer RNAs, small RNA fractions (under $150 \mathrm{nt}$ in size) were prepared as described (Maréchal-Drouard et al., 1995). In brief, RNA was incubated in a $2 \mathrm{M}$ lithium chloride solution over-night at $4^{\circ} \mathrm{C}$. After centrifugation at $16000 \mathrm{~g}$ for $30 \mathrm{~min}$ at $4^{\circ} \mathrm{C}$, the supernatants containing the tRNAs were recovered. Transfer RNAs were thereafter precipitated with 0.1 volume of $1 \mathrm{M}$ sodium acetate $\mathrm{pH} 4.5$ solution and 2.5 volumes of ethanol over-night at $-20^{\circ} \mathrm{C}$. Pelleted tRNAs were dissolved in water and loaded on $15 \%$ polyacrylamide with $7 \mathrm{M}$ Urea and $1 \mathrm{X}$ TBE gels. After Ethidium bromide staining, gel slices were cut from the gels. The tRNAs were eluted over-night at room temperature in a solution composed of $0.5 \mathrm{M}$ ammonium acetate, $10 \mathrm{mM}$ magnesium acetate, $0.1 \mathrm{mM}$ EDTA and $0.1 \%$ SDS. After phenol extraction, tRNAs were ethanol precipitated and finally recovered in $10 \mu \mathrm{l}$ water.

\section{Microbeads-Coupled tDNA Probes for tRNA Isolation}

To purify tRNA molecules, we used the microMACS Streptavidin MicroBeads Kit (Miltenyi Biotec). We synthesized 37 nt long $3^{\prime}$-biotinylated DNA probes complementary to the $3^{\prime}$ half of the C. elegans tRNAs. Oligonucleotide sequences of the tDNA probes were deduced from the C. elegans tRNA genes predictions (GtRNAdb, Lowe lab, Biomolecular Engineering, University of California Santa Cruz). The sources of RNA from which specific tRNAs were isolated were either the whole small RNA populations or the gel-eluted tRNA fractions showed in Figure 3. The procedure was as recommended by the microMACS Kit, except the annealing buffer was made of $10 \mathrm{mM}$ Tris $\mathrm{HCl} \mathrm{pH}$ 7.5, $5 \mathrm{mM} \mathrm{MgCl2}$. Binding/Wash buffer $5 \mathrm{X}$ was $50 \mathrm{mM}$ Tris $\mathrm{HCl} \mathrm{pH} 7.5,5 \mathrm{mM}$ EDTA $\mathrm{pH}$, and $2.5 \mathrm{M} \mathrm{NaCl}$, as indicated. Elutions from microbeads were in $200 \mu \mathrm{l} \mathrm{TE}$. For each feeding and behavioral assay, we used $10 \mu \mathrm{l}$ eluate per worm culture dish, as described in the section "RNA-Feeding" below.

\section{High Purification of tRNA Molecules After Affinity Chromatography and 3' pCp-Cy3 Labeling}

To obtain highly purified Alanine tRNA (UGC) molecules, we used the Streptavidin Sepharose ${ }^{\mathrm{TM}}$ High Performance beads (GE Healthcare, 17-5113-01) coupled to the 3'-biotinylated tDNA Ala (TGC)-3' probe (probe Ala TGC N ${ }^{\circ} 3$, as described).

We first mixed 10-20 $\mu \mathrm{g}$ of total tRNA with 1 nmole of tDNA probe and $40 \mathrm{U}$ of RNaseOUT in $500 \mu \mathrm{l}$ of annealing buffer [5X SSC (saline-sodium citrate); 0.05\% SDS (sodium dodecyl sulfate)]. The mix was then incubated sequentially, $5 \mathrm{~min}$ at 
$70^{\circ} \mathrm{C}, 30 \mathrm{~s}$ at $4^{\circ} \mathrm{C}$ and $2.5 \mathrm{~h}$ at $42^{\circ} \mathrm{C}$. Twenty $\mu \mathrm{l}$ of Streptavidin Sepharose beads were deposited in a $20 \mu \mathrm{m}$ receiver column (Macherey-Nagel, 740522) and equilibrated with $3 \times 500 \mu l$ of annealing buffer. Columns were incubated $15 \mathrm{~min}$ at room temperature in gentle shaking and $15 \mathrm{~min}$ at $42^{\circ} \mathrm{C}$. They were centrifuged $15 \mathrm{~s}$ at $11000 \mathrm{~g}$ and then washed as follows: $2 \times 500 \mu \mathrm{l}$ of $5 \mathrm{x} \mathrm{SSC}\left(5 \mathrm{~min}\right.$ at $\left.42^{\circ} \mathrm{C}\right), 1 \times 500 \mu \mathrm{l} 2 \mathrm{x}$ SSC $(5 \mathrm{~min}$ at $\left.42^{\circ} \mathrm{C}\right), 1 \times 500 \mu \mathrm{l} 2 \mathrm{x} \mathrm{SSC}\left(15 \mathrm{~min}\right.$ at $\left.47^{\circ} \mathrm{C}\right), 1 \times 500 \mu \mathrm{l} 2 \mathrm{x}$ SSC $\left(15 \mathrm{~min}\right.$ at $\left.52^{\circ} \mathrm{C}\right)$. After each washing step, the column was centrifuged $15 \mathrm{sec}$ at $11000 \mathrm{~g}$. tRNA elution was by adding $2 \times 300 \mu \mathrm{l}$ of elution buffer $(10 \mathrm{mM}$ Tris- $\mathrm{HCl} \mathrm{pH} \mathrm{7.5;1} \mathrm{mM}$ EDTA; $5 \mathrm{M}$ Urea). Total elution was ethanol precipitated and resuspended in $2.96 \mu \mathrm{l}$ of water for $3^{\prime} \mathrm{pCp}-\mathrm{Cy} 3$ (Jena Bioscience NU-1706-CY3) labeling.

Before labeling, we added 26\% (v/v) DMSO (1,04 $\mu \mathrm{l})$ to the purified tRNA $(2,96 \mu \mathrm{l})$ and incubated $10 \mathrm{~s}$ at $100^{\circ} \mathrm{C}$ and immediately cooled on ice. The labeling reaction was performed using the Biolabs T4 RNA ligase (M0204). Ligase buffer and ATP were added to the tRNA-DMSO mix and ligation was performed with $20 \mu \mathrm{M}$ of pCp-Cy3 and 5 units of T4 RNA ligase in $10 \mu \mathrm{l}$ at $16^{\circ} \mathrm{C}$ overnight. The labeling was done in the same way for total tRNAs fraction, using $2.5 \mu \mathrm{g}$ total tRNAs.

The different fractions were analyzed by electrophoresis on 7 M Urea-15\% acrylamide gel and scanned on a GE Healthcare Ettan DIGE imager system. The bands corresponding to the purified tRNA were cut from the gel and eluted overnight at room temperature in $0.5 \mathrm{M}$ ammonium acetate, $10 \mathrm{mM}$ magnesium acetate, $0.1 \mathrm{mM}$ EDTA and $0.1 \%$ SDS. After phenol extraction, $\mathrm{tRNAs}$ were ethanol precipitated and finally recovered in $8 \mu \mathrm{l}$ of water.

\section{Northern Blots Analyses}

Northern blots were performed as described in Cognat et al. (2017).

Roughly, RNAs fractionated on a denaturing $7 \mathrm{M}$ Urea/15\% polyacrylamide gel were transferred onto Hybond$\mathrm{N}+$ membrane (Amersham Pharmacia Biotech). Membranes were then hybridized with ${ }^{32} \mathrm{P}$ labeled probes specific to C. elegans tRNAs:

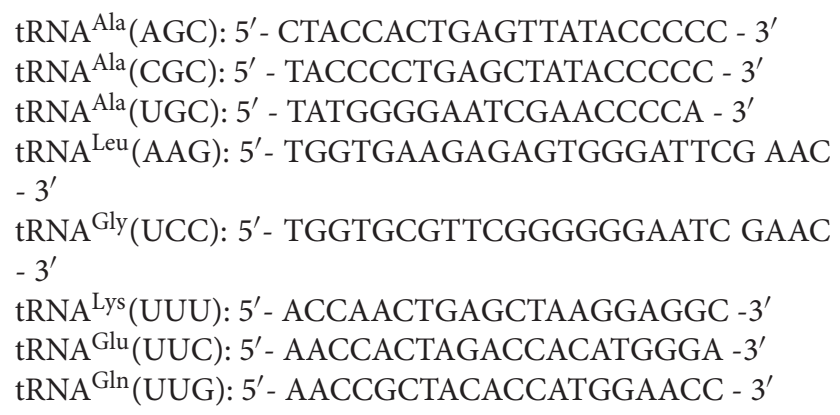

\section{RNA-Feeding}

NGM agar plates were loaded with $40 \mu \mathrm{l}$ of OP50 culture, which, after drying, formed approximately a $100 \mathrm{~mm}^{2}$ spot. Volumes of $10 \mu \mathrm{l}$ RNA to be assayed were deposited per E. coli spot and shortly dried. Synchronized naive embryos were spawned on the RNA-loaded plates, and worms were grown at $20^{\circ} \mathrm{C}$ until adulthood. For imprinting inheritance after multi-generational tRNA feeding (Figure 5), N2 worms were grown from the spawn embryo to the adult laying stage on 1 ng CI-tRNAloaded culture plates (F1). Part of the next generation was grown on new CI-tRNA-loaded culture plates - the second tRNA-fed generation F2 -, another on a regular plate without CI-tRNA - the first naive generation $\mathrm{F} 1+1$. F3 is the progeny of F2 grown on tRNA while $\mathrm{F} 2+1$ is the progeny of $\mathrm{F} 2$ grown without tRNA. The CI chemo-response of the naive generations from the seven successive tRNA fed generations (F1 to F7) was determined as described.

\section{tDNA Oligonucleotides Probes Used for Microbeads tRNAs Purification}

(1) Ala AGC: $5^{\prime}$ TGGAGGTTTGGGGAATTGAACCCCAGC CСТCTCCCAT $3^{\prime}$

(2) Ala CGC: $5^{\prime}$ TGGAGGCACGGGGGATTGAACCCCGGA CTTCCCGCAT $3^{\prime}$

(3) Ala TGC: $5^{\prime}$ TGGAGgTATGGGGAATCGAACCCCAG ACTTCTCGCAT $3^{\prime}$

(4) Ala TGC: 5' ATGCAAAgCCAGCGCTCTACCCCTGAG CTATACCCCC $3^{\prime}$

(5) Arg ACG: 5' CGACCACGGCAGGATTCGAACCTACAA TCTTCTGC $3^{\prime}$

(6) Arg CCG: $5^{\prime}$ AGCTCGCGGAGGGACTTGAACCCCCA TTCCCGGTTCC $3^{\prime}$

(7) Arg CCT: 5' CGACCGAGGCAGGACTCGAACCTGCTG TCTTCGGTTT $3^{\prime}$

(8) Asn GTT: $5^{\prime}$ CGCTCCCGGTGGGCTCGAATCACCTT TCGGTTAA $3^{\prime}$

(9) Asp GTC: $5^{\prime}$ CTCCCCGGCCGGGAATTGAACCCGGGT CTCGCATGTG $3^{\prime}$

(10) Cys GCA: 5' CTAGCTCTCCAGGGACCAAGTTGAGGC CCACGGGGGA $3^{\prime}$

(11) Gln CTG: 5' CTTAGGACGCTGGGCTCAAGTTTAGA GCCACCCTGGA $3^{\prime}$

(12) Gln TTG: 5' CTTAGGACGCTGGGCTCAAGTTTAGAG CCACCTTGGA $3^{\prime}$

(13) Glu CTC: 5' GTGGGTATTCCGGCCCCAAGCTAAGGG GCGTTGCTTT $3^{\prime}$

(14) Glu TTC: 5' GTGGGTGCGCCGGGCCCAAGCTAAGGG CCGTACCCTT $3^{\prime}$

(15) Gly CCC: $5^{\prime}$ ACCATTGTCTCGCGCCCAAGCTTAGGG CAGGTGGCGT $3^{\prime}$

(16) Gly TCC: 5' GTTCGTAAGCTGCCCCCAAGCTAAGGG GAGCTTGCGT $3^{\prime}$

(17) His GTG: 5' CCGGCACCGCTGCGACCAAGCTAAGGT CGTCGTCCGT $3^{\prime}$

(18) Ile AAT: $5^{\prime}$ TTCGGGTTCCAGCGTCCAAGCTGGGGAC GACCGCCGT $3^{\prime}$

(19) Ile TAT: 5' CAATTTGGTCAGCGCCCAAGCTTAGGGC GGGCCCCGT $3^{\prime}$

(20) Leu AAG: 5' GGGAAGCCCCCGCACCCAAGCTTAGG GAGAGAGAAGT $3^{\prime}$

(21) Leu CAA: 5'AGCATACCCACGCACCCAAGCTTAGGGT GAAGCACGT $3^{\prime}$ 
(22) Leu CAG: 5'AGAGGCCTCCCGCGTCCAAGCTTAGGAC GCCTGCCGT $3^{\prime}$

(23) Leu TAA: 5' GGGAGGCCCCCGCACCCAAGCTTAGGG TGAGAGTAGT $3^{\prime}$

(24) Lys CTT: 5' ATTAGACCAACAGCGCCAAAGCTCGGGG CGTAACCCA $3^{\prime}$

(25) Lys TTT: 5' TTAGAATTCCAGTCCCCAAGCTCAGGGG ATCCACCGA $3^{\prime}$

(26) Met CAT: $5^{\prime}$ TTGGGTCTCCAGCCACCTAGCTTTGGTG AGCGACGAT $3^{\prime}$

(27) Met CAT: $5^{\prime}$ TTAGACTTCCAGCACTCAAGCTCGGAGT GGCCCTCGT 3

(28) Phe GAA: 5' TTtagCAatCCAGTgGtCAAgCTAGG ACCAAGCCCGT $3^{\prime}$

(29) Pro AGG: 5' CACGTtCTCTAGgGCCCAAGCTAGGGG CCAAGCTGGG $3^{\prime}$

(30) Pro CGG: $5^{\prime}$ CACGCTCTCCAGGGCCCAAGCTAAGGG CCAAGCCGGG $3^{\prime}$

(31) Pro TGG: 5' CACGCTCTCCAGGGACCAAGTTAGGG GCCAAGCCGGG $3^{\prime}$

(32) Ser AGA: $5^{\prime}$ CCGAGACGGGCGCATCCAAGCTTAGG ACGACTGACGC $3^{\prime}$

(33) Ser CGA: 5' CCGAGACGGGCGCATCCAAGCTTAGG ACGACTGACGC $3^{\prime}$

(34) Ser GCT: 5' CCCAAAGGGGCGCACTCAAGCTTAGAG TAGAACTAGC $3^{\prime}$

(35) Thr AGT: 5' TTTGTCTTCCAGCGACCAAGCTAAgGT CGTACTCCGT $3^{\prime}$

(36) Thr CGT: 5' TTTGTCTTCCAGCTGCCAAGTTAGGGC AGACCCCCGT $3^{\prime}$

(37) Thr TGT: $5^{\prime}$ TAGTTATCCAGGCCCCAAGCTGGGGAG CATTCCCAGT $3^{\prime}$

(38) Trp CCA: 5' CTAGCTTTCCATCCCGCAAGCTAGGCG AGTCACCAGT $3^{\prime}$

(39) Tyr GTA: $5^{\prime}$ TAGgAatCCAGTGACCAAGCTTAGGCC AAGCTGCCT $3^{\prime}$

(40) Val AAC: $5^{\prime}$ TGTGTCTTCCAGCCACCAAGCTCGGGC GGGCTCTAGT $3^{\prime}$

(41) Val CAC: $5^{\prime}$ TGCGTCTTCCAGCGGCCAAGCTTGGGC CGGTCCTGGA $3^{\prime}$

(42) Val TAC: $5^{\prime}$ TGCGTCTTCTAGCGGCCAAGCTTGGGCC GATCCTGGA $3^{\prime}$

\section{DATA AVAILABILITY STATEMENT}

The original contributions presented in the study are included in the article/Supplementary Material, further inquiries can be directed to the corresponding author/s.

\section{AUTHOR CONTRIBUTIONS}

LD and J-JR initiated the project and designed the experimental strategy. DF and J-JR designed and performed the C. elegans experiments: tRNA feeding, chemotaxis tests, statistics. TS-G and $\mathrm{LD}$ performed the biochemical fractionation of RNA and Northern analysis. LD, TS-G, DF, and J-JR analyzed the data and wrote the manuscript. DF designed the figures. All authors contributed to the article and approved the submitted version.

\section{FUNDING}

This work was supported by a grant of the "Agence Nationale de la Recherche" ANR-12-Bioadapt-0022, and by the LABEX ANR-11-LABX-0057_MITOCROSS program. This manuscript has been released as a pre-print at bioRxiv: Fernandes De Abreu et al. (2020).

\section{ACKNOWLEDGMENTS}

We thank Craig Hunter for the sid-2 (qt13) mutant. The elongator mutants elpc-1 (ng10) or ikap-1 (ng10) and elpc-3 (tm3120) were obtained thanks to Jachen A. Solinger via Grazia Malabarba. We also thank Sebastian Leidel for providing the tut-1 (tm1297) worms, and Shohei Mitani for the elpc-1 (tm2149) and elpc1 (tm11684) deletion mutants. We also thank Wormbase for providing valuable databases. Thanks to V. Grandjean and G. Raad for stimulating discussions and incentive suggestions.

\section{SUPPLEMENTARY MATERIAL}

The Supplementary Material for this article can be found online at: https://www.frontiersin.org/articles/10.3389/fcell.2020. 571359/full\#supplementary-material

Supplementary Figure 1 | A specific population of small RNA transfers olfactory imprinting. (a) RNA from BA-exposed and unexposed naive was fractionated into two populations: the larger than 200 nucleotides fraction (Large-RNA) and the smaller than 200 nucleotides fraction (Small-RNA). Worms fed on $10 \mathrm{ng}$ of Large BA-RNA migrate in a BA gradient as worms fed on $10 \mathrm{ng}$ of Large Naive-RNA. By contrast, worms fed on $10 \mathrm{ng}$ of Small BA-RNA display enhanced BA responses, compared to worms fed on $10 \mathrm{ng}$ of Small Naive-RNA or to worms fed on $10 \mathrm{ng}$ of Large BA-RNA (experimental repeats $>4,{ }^{* * *} p$-value $<0.001$ ). (b) $3.5 \%$ low-melting agarose gels fractionate BA-RNA and Naive-RNA into five A to $E$ discrete bands (left side of insert). Naive worms were fed on each of the five gel-purified NA-RNA and BA-RNA populations. Compared BA 1/300 MMI shows that only the BA-RNA band $D$ is able to transfer a BA-imprint to naive (experimental repeats $>4,{ }^{* * *} p$-value $<0.001$ ). (L), NEB double stranded RNA ladder.

Supplementary Figure 2 | Wild-type tRNA Ala UGC has no effect on elpc-1 mutant worms chemo-attraction. Compared chemo-attractive responses of naive or of wild-type tRNAAla UGC fed elpc-1 (tm2149) and elpc-1 (tm11684) mutant worms. Mean Migration Indices (MMI) in gradients of Citronellol (Cl), Benzaldehyde (BA) at the indicated dilutions, were determined as described (experimental repeats $\geq 4,{ }^{* *} p$-value $<0.01$ ).

Supplementary Figure Method 1 | Schematic outline of the chemotaxis assay. Chemotaxis assays were performed on square plates in order to index the worms' positions between the starting line (time 0 , position $0 \mathrm{~cm}$ ) and the odor source (position $+6 \mathrm{~cm}$ ) or water source (position $-6 \mathrm{~cm}$ ). $3 \mathrm{ml}$ of odor solution or water are added to the lids of the plates. 20 synchronized worms are placed at the starting line at time 0 . Worms start their migration and their position is carefully noted, usually at four time-points, at 10, 20, 30 and 40 min past time 0 . Worms that migrate up the odor gradients are positively indexed (from 0 to 6 ). Worms that migrate down the odor gradients toward water are negatively indexed (from 0 to 6). The mean value of all indexed positions at all noted times (in $\mathrm{cm}$ from the starting line) of each of the 20 worms involved in the assay is the Mean Migration Index (MMI). 


\section{REFERENCES}

Agris, P. (2015). The importance of being modified: an unrealized code to RNA structure and function. RNA 21, 552-554. doi: 10.1261/rna.050575. 115

Armelin-Correa, L. M., Gutiyama, L. M., Brandt, D. Y., and Malnic, B. (2014). Nuclear compartmentalization of odorant receptor genes. Proc. Natl. Acad. Sci. U.S.A. 111, 2782-2787. doi: 10.1073/pnas.1317036111

Avihu, H. Z., Bloom-Ackermann, Z., Frumkin, I., Hanson-Smith, V., CharpakAmikam, Y., Feng, Q. H., et al. (2013). tRNA genes rapidly change in evolution to meet novel translational demands. eLife 2:e01339.

Bargmann, C. I. (2006). Chemosensation in C. elegans. WormBook 25, 1-29. doi: 10.1895/wormbook.1.123.1

Bargmann, C. I., Hartwieg, E., and Horvitz, H. R. (1993). Odorant-selective genes and neurons mediate olfaction in C. elegans. Cell 74, 515-527. doi: 10.1016/ 0092-8674(93)80053-h

Benito, E., Kerimoglu, C., Ramachandran, B., Pena-Centeno, T., Jain, G., Stilling, R. M., et al. (2018). RNA-dependent intergenerational inheritance of enhanced synaptic plasticity after environmental enrichment. Cell Rep. 23, 546-554. doi: 10.1016/j.celrep.2018.03.059

Boccaletto, P., Machnicka, M. A., Purta, E., Piątkowski, P., Bagiński, B., Wirecki, T. K., et al. (2018). MODOMICS: a database of RNA modification pathways. 2017 update. Nucleic Acids Res. 46, D303-D307.

Boussard, A., Delescluze, J., Perez-Escudero, A., and Dussutour, A. (2019). Memory inception and preservation in slime moulds: the quest for a common mechanism. Philos. Trans. R. Soc. B Biol. Sci. 374:20180368. doi: 10.1098/rstb. 2018.0368

Chan, P. P., and Lowe, T. M. (2009). GtRNAdb: a database of transfer RNA genes detected in genomic sequence. Nucleic Acids Res. 37, D93-D97.

Chen, C. C., Tuck, S., and Byström, A. S. (2009). Defects in tRNA modification associated with neurological and developmental dysfunctions in Caenorhabditis elegans elongator mutants. PLoS Genet. 5:e1000561. doi: 10.1371/journal.pgen. 1000561

Cognat, V., Morelle, G., Megel, C., Lalande, S., Molinier, J., Vincent, T., et al. (2017). The nuclear and organellar tRNA-derived RNA fragment population in Arabidopsis thaliana is highly dynamic. Nucleic Acids Res. 45, 3460-3472. doi: 10.1093/nar/gkw1122

Cozen, A. E., Quartley, E., Holmes, A. D., Hrabeta-Robinson, E., Phizicky, E. M., and Lowe, T. M. (2015). ARM-seq: AlkB-facilitated RNA methylation sequencing reveals a complex landscape of modified tRNA fragments. Nat. Methods 12, 879-884. doi: 10.1038/nmeth.3508

Creppe, C., Malinouskaya, L., Volvert, M. L., Gillard, M., Close, P., Malaise, O., et al. (2009). Elongator controls the migration and differentiation of cortical neurons through acetylation of alpha-tubulin. Cell 136, 551-564. doi: 10.1016/ j.cell.2008.11.043

Dalwadi, U., and Yip, C. K. (2018). Structural insights into the function of Elongator. Cell. Mol. Life Sci. 75, 1613-1622. doi: 10.1007/s00018-0182747-6

Dauden, M. I., Jaciuk, M., Weis, F., Lin, T. Y., Kleindienst, C., Abbassi, N. E. H., et al. (2019). Molecular basis of tRNA recognition by the Elongator complex. Sci. Adv. 5:eaaw2326. doi: 10.1126/sciadv.aaw2326

Dauden, M. I., Kosinski, J., Kolaj-Robin, O., Desfosses, A., Ori, A., Faux, C., et al. (2017). Architecture of the yeast Elongator complex. EMBO Rep. 18, 264-279. doi: $10.15252 / \mathrm{embr} .201643353$

Degl'Innocenti, A., and D'Errico, A. (2017). Regulatory features for odorant receptor genes in the mouse genome. Front. Genet. 8:19. doi: 10.3389/fgene. 2017.00019

Deng, W., Babu, R., Su, D., Yin, S., Begley, T. J., and Dedon, P. C. (2015). Trm9-catalyzed tRNA modifications regulate global protein expression by codon-biased translation. PLoS Genet. 11:e1005706. doi: 10.1371/journal.pgen. 1005706

Devanapally, S., Ravikumar, S., and Jose, A. M. (2015). Double-stranded RNA made in C. elegans neurons can enter the germline and cause transgenerational gene silencing. Proc. Natl. Acad. Sci. U.S.A. 112, 2133-2138. doi: 10.1073/pnas. 1423333112

Dittman, A. H., and Quinn, T. P. (1996). Homing in pacific salmon mechanisms and ecological basis. J. Exp. Biol. 199, 83-91.
Duechler, M., Leszczyńska, G., Sochacka, E., and Nawrot, B. (2016). Nucleoside modifications in the regulation of gene expression: focus on tRNA. Cell. Mol. Life Sci. 73, 3075-3095. doi: 10.1007/s00018-016-2217-y

Duret, L. (2000). tRNA gene number and codon usage in the C. elegans genome are co-adapted for optimal translation of highly expressed genes. Trends Genet. 16, 287-289. doi: 10.1016/s0168-9525(00)02041-2

Engelke, D. R., and Hopper, A. K. (2006). Modified view of tRNA: stability amid sequence diversity. Mol. Cell 21, 144-145. doi: 10.1016/j.molcel.2006.01.002

Evans, K. J., Huang, N., Stempor, P., Chesney, M. A., Down, T. A., and Ahringer, J. (2016). Stable Caenorhabditis elegans chromatin domains separate broadly expressed and developmentally regulated genes. Proc. Natl. Acad. Sci. U.S.A. 113, E7020-E7029. doi: 10.1073/pnas.1608162113

Fernandes De Abreu, D. A., Salinas-Giegé, T., Drouard, L., and Remy, J. J. (2020). Alanine tRNA translate environment into behavior in Caenorhabditis elegans. bioRxiv [Preprint] doi: 10.1101/289629

Gapp, K., Jawaid, A., Sarkies, P., Bohacek, J., Pelczar, P., Prados, J., et al. (2014). Implication of sperm RNAs in transgenerational inheritance of the effects of early trauma in mice. Nat. Neurosci. 17, 667-669. doi: 10.1038/nn.3695

Gecse, E., Gilányi, B., Csaba, M., Hadju, G., and Soti, C. (2019). A cellular defense memory imprinted by early life toxic stress. Sci. Rep. 9:18935. doi: 10.1038/ s41598-019-55198-4

Gerlach, G., Atema, J., Kingsford, M. J., Black, K. P., and Miller-Sims, V. (2007). Smelling home can prevent dispersal of reef fish larvae. Proc. Natl. Acad. Sci. U.S.A. 104, 858-863. doi: 10.1073/pnas.0606777104

Gerlach, G., Tietje, K., Biechl, D., Namekawa, I., Schalm, G., and Sulmann, A. (2019). Behavioural and neuronal basis of olfactory imprinting and kin recognition in larval fish. J. Exp. Biol. 222:jeb189746. doi: 10.1242/jeb.189746

Glatt, S., Letoquart, J., Faux, C., Taylor, N. M., Seraphin, B., and Muller, C. W. (2012). The Elongator subcomplex Elp456 is a hexameric RecA-like ATPase. Nat. Struct. Mol. Biol. 19, 314-320. doi: 10.1038/nsmb.2234

Grandjean, V., Fourré, S., De Abreu, D. A. F., Derieppe, M. A., Remy, J. J., and Rassoulzadegan, M. (2015). RNA-mediated paternal heredity of diet-induced obesity and metabolic disorders. Sci. Rep. 5:18193. doi: 10.1038/srep18193

Grassman, M. A., Owens, D. W., Mcvey, J. P., and Marquez, M. (1984). Olfactorybased orientation in artificially imprinted sea turtles. Science 224, 83-84. doi: 10.1126/science.224.4644.83

Hall, S. E., Beverly, M., Russ, C., Nusbaum, C., and Sengupta, P. (2010). A cellular memory of developmental history generates phenotypic diversity in C. elegans. Curr. Biol. 20, 149-155. doi: 10.1016/j.cub.2009.11.035

Hall, S. E., Chirn, G.-W., Lau, N. C., and Sengupta, P. (2013). RNAi pathways contribute to developmental history-dependent phenotypic plasticity in C. elegans. RNA 19, 306-319. doi: 10.1261/rna.036418.112

Hart, A. C., and Chao, M. Y. (2010). "From odors to behaviors in Caenorhabditis elegans," in The Neurobiology of Olfaction, ed. A. Menini (Boca Raton, FL: CRC Press).

Hong, M., Ryu, L., Ow, M. C., Kim, J., Je, A. R., Chinta, S., et al. (2017). Early pheromone experience modifies a synaptic activity to influence adult pheromone responses of C. elegans. Curr. Biol. 27, 3168-3177. doi: 10.1016/j. cub.2017.08.068

Horn, G. (2004). Pathways of the past: the imprint of memory. Nat. Rev. Neurosci. 5, 108-120. doi: 10.1038/nrn1324

Jablonka, E., and Lamb, M. J. (2015). The inheritance of acquired epigenetic variations. Int. J. Epidemiol. 44, 1094-1103. doi: 10.1093/ije/dyv020

Jin, X., Pokala, N., and Bargmann, C. I. (2016). Distinct circuits for the formation and retrieval of an imprinted olfactory memory. Cell 164, 632-643. doi: 10.1016/ j.cell.2016.01.007

Jonkhout, N., Tran, J., Smith, M. A., Schonrock, N., Mattick, J. S., and Novoa, E. M. (2017). The RNA modification landscape in human desease. RNA 23, 1754-1769. doi: 10.1261/rna.063503.117

Jose, A. M., Smith, J. J., and Hunter, C. P. (2009). Export of RNA silencing from C. elegans tissues does not require the RNA channel SID-1. Proc. Natl. Acad. Sci. U.S.A. 106, 2283-2288. doi: 10.1073/pnas.0809760106

Juang, B. T., Gu, C., Starnes, L., Palladino, F., Goga, A., Kennedy, S., et al. (2013). Endogenous nuclear RNAi mediates behavioral adaptation to odor. Cell 154, 1010-1022. doi: 10.1016/j.cell.2013.08.006

Karlsborn, T., Tükenmez, H., Mahmud, A. K., Xu, F., Xu, H., and Byström, A. S. (2014). Elongator, a conserved complex required for wobble uridine 
modifications in eukaryotes. RNA Biol. 11, 1519-1528. doi: 10.4161/15476286. 2014.992276

Kawamura, K., and Maruyama, I. N. (2019). Forward genetic screen for Caenorhabditis elegans mutants with a shortened locomotor healthspan. G3 9, 2415-2423. doi: 10.1534/g3.119.400241

Kirchner, S., and Ignatova, Z. (2015). Emerging roles of tRNA in adaptive translation, signaling dynamics and disease. Nat. Rev. Genet. 16, 641-652.

Kirkland, J. G., and Kamakaka, R. T. (2015). tRNA insulator function: insight into inheritance of transcriptional states? Epigenetics 5, 96-99. doi: 10.4161/epi.5. 10775

Kojic, M., and Wainwright, B. (2016). The many faces of elongator in neurodevelopment and disease. Front. Mol. Neurosci. 9:115. doi: 10.3389/fnmol. 2016.00115

Larsen, A. T., Fahrenbach, A. C., Sheng, J., Pian, J., and Szostak, J. W. (2015). Thermodynamic insights into 2-thiouridine-enhanced RNA hybridization. Nucleic Acids Res. 43, 7675-7687. doi: 10.1093/nar/gkv761

Liberman, L., Wang, S. Y., and Greer, E. L. (2019). Transgenerational epigenetic inheritance: from phenomenon to molecular mechanisms. Curr. Opin. Neurobiol. 59, 189-206. doi: 10.1016/j.conb.2019.09.012

Maréchal-Drouard, L., Small, I., Weil, J. H., and Dietrich, A. (1995). Transfer RNA import into plant mitochondria. Methods Enzymol. 260, 310-327. doi: 10.1016/0076-6879(95)60148-1

Marré, J., Traver, E. C., and Jose, A. M. (2016). Extracellular RNA is transported from one generation to the next in Caenorhabditis elegans. Proc. Natl. Acad. Sci. U.S.A. 113, 12496-12501. doi: 10.1073/pnas.1608959113

McEwan, D. L., Weisman, A. S., and Hunter, C. P. (2012). Uptake of extracellular double-stranded RNA by SID-2. Mol. Cell 47, 746-754. doi: 10.1016/j.molcel. 2012.07.014

McFarlane, R. J., and Whitehall, S. K. (2009). tRNA genes in eukaryotic genome organization and reorganization. Cell Cycle 19, 3102-3106. doi: 10.4161/cc.8. 19.9625

Megel, C., Morelle, G., Lalande, S., Duchêne, A. M., Small, I., and MarechalDrouard, L. (2015). Surveillance and cleavage of eukaryotic tRNAs. Int. J. Mol. Sci. 16, 1873-1893. doi: 10.3390/ijms16011873

Motorin, Y., and Helm, M. (2019). Methods for RNA modification mapping using deep sequencing: established and new emerging technologies. Genes 10:35. doi: 10.3390/genes10010035

Nedialkova, D. D., and Leidel, S. A. (2015). Optimization of codon translation rates via tRNA modifications maintains proteome integrity. Cell 161, 1606-1618. doi: 10.1016/j.cell.2015.05.022

O’Halloran, D. M., Altshuler-Keylin, S., Lee, J. I., and L'Etoile, N. D. (2009). Regulators of AWC-mediated olfactory plasticity in Caenorhabditis elegans. PLoS Genet. 5:e1000761. doi: 10.1371/journal.pgen.1000761

Okada, Y., Yamagata, K., Hong, K., Wakayama, T., and Zhang, Y. (2010). A role for the elongator complex in zygotic paternal genome demethylation. Nature 463, 554-558. doi: 10.1038/nature 08732

O’Rourke, S. M., Dorfman, M. D., Carter, J. C., and Bowerman, B. (2007). Dynein modifiers in C. elegans: light chains suppress conditional heavy chain mutants. PLoS Genet. 3:e128. doi: 10.1371/journal.pgen.0030128

Pang, J. Y. L., Abo, R., Levine, S. S., and Dedon, P. (2014). Diverse cell stresses induce unique patterns of tRNA up- and down-regulation: tRNA-seq for quantifying changes in tRNA copy number. Nucleic Acids Res. 42:e170. doi: 10.1093/nar/gku945

Pliatsika, V., Loher, P., Magee, R., Telonis, A. G., Londin, E., Shigematsu, M., et al. (2018). MINTbase v2.0: a comprehensive database for tRNA-derived fragments that includes nuclear and mitochondrial fragments from all The Cancer Genome Atlas projects. Nucleic Acids Res. 46, D152-D159. doi: 10.1093/ nar/gkx1075

Posner, R., Toker, I. A., Antonova, O., Star, E., Anava, S., Azmon, E., et al. (2019). Neuronal small RNAs control behavior transgenerationally. Cell 177, 1814-1826.e15. doi: 10.1016/j.cell.2019.04.029

Pratt-Hyatt, M., Pai, D. A., Haeusler, R. A., Wozniak, G. G., Good, P. D., Miller, E. L., et al. (2013). Mod5 protein binds to tRNA gene complexes and affects local transcriptional silencing. Proc. Natl. Acad. Sci. U.S.A. 110, E3081-E3089. doi: 10.1073/pnas.1219946110

Raab, J. R., Chiu, J., Zhu, J. C., Katzman, S., Kurukuti, S., Wade, A. A., et al. (2012). Human tRNA genes function as chromatin insulators. EMBO J. 31, 330-350. doi: 10.1038/emboj.2011.406

Rechavi, O., Houri-Ze'evi, L., Anava, S., Goh, W. S., Kerk, S. Y., and Hobert, O. (2014). Starvation-induced transgenerational inheritance of small RNAs in C. elegans. Cell 158, 277-287. doi: 10.1016/j.cell.2014.06.020

Remy, J. J. (2010). Stable inheritance of an acquired behavior in C. elegans. Curr. Biol. 20, 877-878. doi: 10.1016/j.cub.2010.08.013

Remy, J. J., and Hobert, O. (2005). An interneuronal chemoreceptor required for olfactory imprinting in C. elegans. Science 309, 787-790. doi: 10.1126/science. 1114209

Robertson, H. M., and Thomas, J. H. (2006). The putative chemoreceptor families of C. elegans. Wormbook 6, 1-12. doi: 10.1895/wormbook.1.66.1

Sarin, L. P., and Leidel, S. A. (2014). Modify or die? - RNA modification defects in metazoans. RNA Biol. 11, 1555-1567. doi: 10.4161/15476286.2014.99 2279

Sauer, P. V., Gu, Y., Liu, W. H., Mattiroli, F., Panne, D., Luger, K., et al. (2018). Mechanistic insights into histone deposition and nucleosome assembly by the chromatin assembly factor-1. Nucleic Acids Res. 46, 9907-9917. doi: 10.1093/ nar/gky823

Schaefer, M., Kapoor, U., and Jantsch, M. F. (2017). Understanding RNA modifications: the promises and technological bottlenecks of the “epitranscriptome." Open Biol. 7:170077. doi: 10.1098/rsob.170077

Schimmel, P. (2018). The emerging complexity of the tRNA world: mammalian tRNAs beyond protein synthesis. Nat. Rev. Mol. Cell Biol. 19, 45-58. doi: 10. 1038/nrm.2017.77

Shigematsu, M., Honda, S., Loher, P., Telonis, A. G., Rigoutsos, I., and Kirino, Y. (2017). YAMAT-seq: an efficient method for high-throughput sequencing of mature transfer RNAs. Nucleic Acids Res. 45:e70.

Solinger, J. A., Paolinelli, R., Klöss, H., Scorza, F. B., Marchesi, S., Sauder, U., et al. (2010). The Caenorhabditis elegans Elongator complex regulates neuronal alpha-tubulin acetylation. PLoS Genet. 6:e1000820. doi: 10.1371/journal.pgen. 1000820

Talbert, P. B., and Henikoff, S. (2009). Chromatin-based transcriptional punctuation. Gen. Dev. 23, 1037-1041. doi: 10.1101/gad.1806409

Tielens, S., Huysseune, S., Godin, J. D., Chariot, A., Malgrange, B., and Nguyen, L. (2016). Elongator controls cortical interneuron migration by regulating actomyosin dynamics. Cell Res. 26, 1131-1148. doi: 10.1038/cr.2016.112

Troemel, E. R., Kimmel, B. E., and Bargmann, C. I. (1997). Reprogramming chemotaxis responses: sensory neurons define olfactory preferences in C. elegans. Cell 91, 161-169. doi: 10.1016/s0092-8674(00)80399-2

Vidal, B., Aghayeva, U., Sun, H., Wang, C., Glenwinkel, L., Bayer, E. A., et al. (2018). An atlas of Caenorhabditis elegans chemoreceptor expression. PLoS Biol. 16:e2004218. doi: 10.1371/journal.pbio.2004218

Woolnough, J. L., Atwood, B. L., and Giles, K. E. (2015). Argonaute 2 binds directly to tRNA genes and promotes gene repression in cis. Mol. Cell. Biol. 35, 2278-2294. doi: 10.1128/mcb.00076-15

Zheng, G., Qin, Y., Clark, W. C., Dai, Q., Yi, C., He, C., et al. (2015). Efficient and quantitative high-throughput tRNA sequencing. 2015. Nat. Methods 12, 835-837. doi: 10.1038/nmeth.3478

Conflict of Interest: The authors declare that the research was conducted in the absence of any commercial or financial relationships that could be construed as a potential conflict of interest.

Copyright $\odot 2020$ Fernandes De Abreu, Salinas-Giegé, Drouard and Remy. This is an open-access article distributed under the terms of the Creative Commons Attribution License (CC BY). The use, distribution or reproduction in other forums is permitted, provided the original author(s) and the copyright owner(s) are credited and that the original publication in this journal is cited, in accordance with accepted academic practice. No use, distribution or reproduction is permitted which does not comply with these terms. 